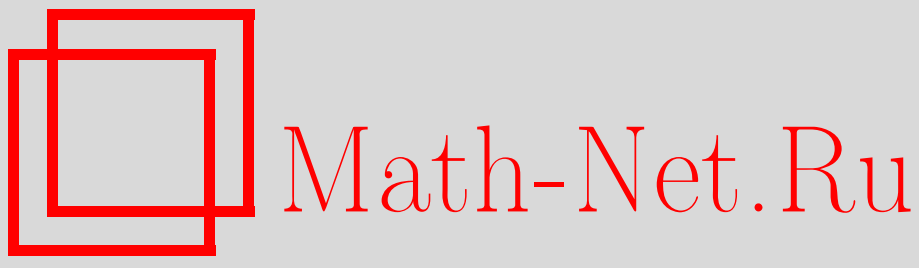

Д. Д. Кармона, П. В. Парамонов, К. Ю. Федоровский, О равномерной аппроксимации полианалитическими многочленами и задаче Дирихле для бианалитических функций, Матем. сб., 2002, том 193, номер 10, 75-98

DOI: https://doi.org/10.4213/sm690

Использование Общероссийского математического портала Math-Net.Ru подразумевает, что вы прочитали и согласны с пользовательским соглашением

http://www . mathnet.ru/rus/agreement

Параметры загрузки:

IP : 54.224 .135 .184

26 апреля 2023 г., 07:01:28 
УДК 517.538.5+517.956.2

Х.Х. Кармона, П.В. Парамонов, К. Ю. Федоровский

\section{О равномерной аппроксимации полианалитическими многочленами и задаче Дирихле для бианалитических функций}

В работе устанавливаются новые необходимые и достаточные условия равномерной приближаемости функций полианалитическими многочленами и полианалитическими рациональными функциями на компактных множествах комплексной плоскости. Анализируется также взаимосвязь между указанными аппроксимационньми задачами и задачей Дирихле для бианалитических функций.

Библиографоия: 23 названия.

\section{$\S 1$. Введение}

Напомним, что функция $f$ назьвается $n$-аналитической на некотором открытом множестве $U \subset \mathbb{C}$, если $\bar{\partial}^{n} f=0$ на $U$ (в классическом смысле). Здесь $n$ натуральное, а

$$
\bar{\partial}=\frac{\partial}{\partial \bar{z}}=\frac{1}{2}\left(\frac{\partial}{\partial x}+i \frac{\partial}{\partial y}\right)
$$

- оператор Коши-Римана. Ясно, что $f$ имеет в $U$ (однозначное) представление

$$
f(z)=\bar{z}^{n-1} f_{n-1}(z)+\cdots+\bar{z} f_{1}(z)+f_{0}(z),
$$

где $f_{0}, \ldots, f_{n-1}$ - голоморфные в $U$ функции. В частности, любой $n$-аналитический полином $p$ (т.е. полином от переменных $z$ и $\bar{z}$, удовлетворяющий всюду в $\mathbb{C}$ уравнению $\left.\bar{\partial}^{n} p=0\right)$ имеет вид:

$$
p(z, \bar{z})=\bar{z}^{n-1} p_{n-1}(z)+\cdots+\bar{z} p_{1}(z)+p_{0}(z),
$$

где $p_{0}, \ldots, p_{n-1}$ - полиномы комплексного переменного $z$. В литературе (см., например, [1]) n-аналитические функции часто называют полианалитическими функциями порядка $n$.

Пусть $X-$ компакт в $\mathbb{C}$ с внутренностью $X^{\circ}, C(X)$ - пространство всех непрерывных на $X$ комплекснозначных функций с равномерной нормой

Работа первого автора поддержана фондом Министерства образования и науки Испании (грант PB98-1242-C02-02) и фондом Генералитета Каталонии (грант 2000-SGR-00059).

Работа второго автора поддержана Российским фондом фундаменталњных исследований (гранты № № 00-01-00618 и 00-15-96008) и фондом Министерства образования и культуры Испании (грант SAB 1998-0049).

Работа третьего автора поддержана Российским фондом фундаментальньт исследований (гранты № № 00-01-00618, 00-15-96008 и 01-01-06373) и фондом INTAS (грант YSF-2001/2-16). 
$\|f\|_{X}=\max _{z \in X}|f(z)|, \mathscr{A}_{n}(X)=\left\{f \in C(X): \bar{\partial}^{n} f=0\right.$ на $\left.X^{\circ}\right\}$. Обозначим через $\mathscr{P}_{n}$ (соответственно $\mathscr{R}_{n}^{X}$ ) совокупность всех $n$-аналитических полиномов (соответственно $n$-аналитических рациональных функций с "полюсами" вне $X$, т.е. функций вида (1.1), у которых все коэффицциенты $f_{0}, \ldots, f_{n-1}$ - рациональные функции от $z$ с полюсами вне $X)$. Определим пространства $\mathscr{P}_{n}(X)$ и $\mathscr{R}_{n}(X, Y)$ (где $Y$ - компакт, содержащий $X$ ) как замыкания в $C(X)$ подпространств $\left\{\left.p\right|_{X}: p \in \mathscr{P}_{n}\right\}$ и $\left\{\left.f\right|_{X}: f \in \mathscr{R}_{n}^{Y}\right\}$ соответственно. Нетрудно показать, что при $1 \leqslant s \leqslant n$ и любом $X$ справедливы включения

$$
\mathscr{P}_{s}(X) \subset \mathscr{P}_{n}(X) \subset \mathscr{R}_{n}(X, X) \subset \mathscr{A}_{n}(X) .
$$

Мы будем изучать следующую задачу аппроксимации для “классов функиий”.

ЗАДАчА 1.1. Найти необходимые и достаточные условия на компакт $X$, при которых выполняется равенство

$$
\mathscr{P}_{n}(X)=\mathscr{A}_{n}(X)
$$

В случае $n=1$ задача 1.1 полностью решена в работе С.Н. Мергеляна [2] в топологических терминах:

Teорема 1.2. Равенство $\mathscr{P}_{1}(X)=\mathscr{A}_{1}(X)$ имеет место тогда и только тогда, когда множество $\mathbb{C} \backslash X$ связно.

Всюду в дальнейшем задача 1.1 рассматривается при фиксированном $n \geqslant 2$. Здесь ситуация оказывается сложнее и интереснее: даже более простая на первый взгляд задача о совпадении пространств $\mathscr{P}_{n}(X)$ и $\mathscr{R}_{n}(X, X)$ до сих пор остается нерешенной (по теореме Рунге $\mathscr{P}_{1}(X)=\mathscr{R}_{1}(X, X)$, если и только если $\mathbb{C} \backslash X$ связно).

В работе [3] доказана следуюшая теорема.

Tеорема 1.3. Пусть $X-$ компакт в $\mathbb{C}$ со связныц дополнением $\mathbb{C} \backslash X$, $n \geqslant 2$. Тогда $\mathscr{P}_{n}(X)=\mathscr{A}_{n}(X)$.

Таким образом, условие связности $\mathbb{C} \backslash X$ является достаточньм в задаче 1.1 и в случае аппроксимации $n$-аналитическими многочленами, $n \geqslant 2$. Однако это условие уже не является необходимым для выполнения (1.2) при $n \geqslant 2$ : если $X-$ замкнутый контур, то соответствующий критерий приближаемости носит "чисто аналитический" характер (см. [4]).

Напомним, что ограниченная область $\Omega$ называется областью Каратеодори, если $\partial \Omega=\partial \Omega_{\infty}$, где $\Omega_{\infty}$ - неограниченная (связная) компонента множества $\overline{\mathbb{C}} \backslash \bar{\Omega}$ (в частности, $\Omega$ односвязна и $\Omega=\bar{\Omega}^{\circ}$ ). Компактное множество $X \subset \mathbb{C}$ называется компактом Каратеодори, если $\partial X=\partial \widehat{X}$, где $\widehat{X}$ - объединение $X$ со всеми ограниченными компонентами его дополнения (так называемая полиномиальная оболочка $X)$.

Под контуром понимается замкнутая жорданова кривая (не обязательно спрямляемая) в $\mathbb{C}$. Если $\Gamma$ - контур, то $D(\Gamma)$ - область, им ограниченная. Пусть $\Gamma_{1}=$ $\{t \in \mathbb{C}:|t|=1\}$ - единичная окружность, а $D_{1}=D\left(\Gamma_{1}\right)=\{w \in \mathbb{C}:|w|<1\}-$ единичный круг.

$\mathrm{B} \S 2$ получены критерий совпадения пространств $C(\partial \Omega)$ и $\mathscr{R}_{n}(\partial \Omega, \bar{\Omega})$ для областей Каратеодори $\Omega$ (теорема $2.2(1))$ и критерий совпадения пространств $\mathscr{A}_{n}(X)$ и 
$\mathscr{P}_{n}(X)$ для компактов Каратеодори $X$ (теорема 2.2(2)). Сушественным для формулировки этих результатов является понятие неванлинновской области (см. определение 2.1).

В $\S 3$ мы исследуем свойства неванлинновских областей и приводим ряд примеров, иллюстрирующих результаты $\S 2$.

В $\S 4$ изучается задача 1.1 в случае $X=\Gamma \cup K$, где $\Gamma$ - контур, а $K$ - компактное подмножество области $D(\Gamma)$. Теорема 4.3 содержит два достаточных условия на $K$ и $Г$, при которых равенство (1.2) выполнено. Далее приводятся примеры, где равенство (1.2) не выполняется и, в частности, показывается, что достаточные условия приближаемости в теореме 4.3 не являются необходимыми. Таким образом, при исследовании задачи 1.1 возникают достаточно сложные аналитические характеристики множества $X$, зависящие также и от $n$. Например, если $\Gamma$ - некоторый эллипс, а $K$ - отрезок, соединяющий его фокусы, то для $X=\Gamma \cup K$ имеет место соотношение $P_{2}(X) \neq P_{3}(X)=C(X)$.

В $\S 5$ (см. следствие 5.3 и теорему 5.5$)$ анализируется взаимосвязь между условием приближаемости (1.2) (где $n=2$, а $X=\partial \Omega$ - гранища жордановой области $\Omega$ ) и условиями разрешимости и единственности в соответствуюшей задаче Дирихле для бианалитических (т.е. 2-аналитических) функций в области $\Omega$. В отличие от классической задачи Дирихле для гармонических функций, до сих пор неизвестно: существуют ли области, регулярные для $\bar{\partial}^{2}$-задачи Дирихле?

\section{§2. Аппроксимация на компактах Каратеодори}

Пусть $\Omega$ - ограниченная односвязная область в $\mathbb{C}, k$ - какое-либо (фиксированное) конформное отображение единичного круга $D_{1}$ на $\Omega$. Известно, что для почти всех (п.в.) $t$ на $\Gamma_{1}$ (относительно длины на $\Gamma_{1}$ ) существуют угловые граничные значения $k(t)$ функции $k$.

ОПРЕДЕЛЕниЕ 2.1. Ограниченная односвязная область $\Omega \subset \mathbb{C}$ называется неванлинновской, если существуют две ограниченные голоморфные функции $u(\cdot)$ и $v(\cdot)$ в $\Omega\left(\right.$ причем $v \not \equiv 0$ ) такие, что для п.в. точек $t \in \Gamma_{1}$ выполняется следуюшее равенство угловых граничных значений:

$$
\overline{k(t)}=\frac{u(k(t))}{v(k(t))}
$$

Это определение корректно, т.е. не зависит от выбора $k$. Более того, по теореме единственности Лузина-Привалова [5; гл. 4 , раздел 2.5$]$ отношение $u / v$ определено в $\Omega$ (для неванлинновской $\Omega$ ) единственным образом.

Класс неванлинновских областей обозначим через $\mathscr{N}$. Используя традиционную терминологию (см., например, [6; гл. II, $\S 6]$ ), можно сказать, что область $\Omega$ является неванлинновской тогда и только тогда, когда сушествуют функции $u, v \in H_{\infty}(\Omega)(v \not \equiv 0)$ такие, что $\bar{\zeta}=u(\zeta) / v(\zeta)$ на $\partial \Omega$ почти всюду (п.в.) в смиссле конформных отображений. Здесь $u(\zeta)$ и $v(\zeta)$ - граничные значения $u(z)$ и $v(z)$ на $\partial \Omega$, существующие на $\partial \Omega$ п.в. в смысле конформных отображений, а через $H_{p}\left(D_{1}\right)$ и $H_{\infty}(\Omega)$ обозначаются соответствуюшие классы Харди, $p \in[1, \infty)$.

Свойства областей класса $\mathscr{N}$ и конкретные примеры таких областей обсуждаются в $\S 3$.

Первым основным результатом настояшей работы является 
ТЕОРема 2.2. Пусть $n \geqslant 2$ - натуральное.

(1) Если $\Omega-$ область Каратеодори в $\mathbb{C}$, то

$$
C(\partial \Omega)=\mathscr{R}_{n}(\partial \Omega, \bar{\Omega}) \Longleftrightarrow \Omega \notin \mathscr{N}
$$

(2) Пусть $X$ - компакт Каратеодори в $\mathbb{C}$. Тогда $\mathscr{A}_{n}(X)=\mathscr{P}_{n}(X)$, если и только если кажсдая ограниченная компонента множества $\mathbb{C} \backslash X$ не является неванлинновской областью.

Всюду в дальнейшем под мерой понимается конечная комплекснозначная борелевская мера в $\mathbb{C}$. Через Spt $\mu$ обозначается носитель меры $\mu$, а через $|\mu|-$ соответствующая ей вариационная мера. Термин “ $\mu$-п.в.", как обычно, означает “ $|\mu|$-п.в." Если $\mu$ - мера, а $E$ - борелевское множество, то $\mu_{\mid E}$ есть сужение меры $\mu$ на множество $E$, т.е. $\mu_{\mid E}\left(E_{1}\right)=\mu\left(E \cap E_{1}\right)$.

Для спрямляемого контура $\Gamma$ через $d \zeta_{\mid \Gamma}$ обозначается мера на $\Gamma$, действующая (как функционал на пространстве $C(\Gamma))$ по формуле $d \zeta_{\mid \Gamma}(f)=\int_{\Gamma} f(\zeta) d \zeta$. В случае $\Gamma=\Gamma_{1}$ мы используем комплексный параметр $t$ (вместо $\zeta$ ) и соответствуюшую меру обозначаем через $d t_{\mid \Gamma_{1}}$.

Преобразованием Коши меры $\mu$ называется (обобшенная) функция

$$
\widehat{\mu}(z)=\frac{1}{2 \pi i} \int \frac{d \mu(\zeta)}{\zeta-z}
$$

Хорошо известно, что $\widehat{\mu}$ голоморфна вне $\operatorname{Spt} \mu$ и $\bar{\partial} \widehat{\mu}=\frac{i}{2} \mu$ в смысле обобшенных функций.

ДоказАТЕЛЬство теоремы 2.2. Докажем (1). Точнее, мы установим, что

$$
C(\partial \Omega) \neq \mathscr{R}_{n}(\partial \Omega, \bar{\Omega}) \Longleftrightarrow \Omega \in \mathscr{N}
$$

Предположим, что $\Omega \in \mathscr{N}$. Тогда найдутся такие голоморфные и ограниченные в $\Omega$ функции $u$ и $v \not \equiv 0$, что вьполняется (2.1). Выберем точку $z_{0} \in \Omega$ такую, что $0<\left|u\left(z_{0}\right)-\bar{z}_{0} v\left(z_{0}\right)\right|$. Покажем, что $\left.\frac{\left(\bar{z}-\bar{z}_{0}\right)^{n-1}}{z-z_{0}}\right|_{\partial \Omega} \notin \mathscr{R}_{n}(\partial \Omega, \bar{\Omega})$. В самом деле, если мы предположим противное, то для любого $\delta>0$ найдутся такие рациональные функции $f_{0}, \ldots, f_{n-1}$ с полюсами вне $\bar{\Omega}$, что

$$
\left|\sum_{s=0}^{n-1} \bar{\zeta}^{s} f_{s}(\zeta)-{\frac{\left(\bar{\zeta}-\bar{z}_{0}\right)}{\zeta-z_{0}}}^{n-1}\right|<\delta
$$

при $\zeta \in \partial \Omega$. Пусть $w_{0}=k^{-1}\left(z_{0}\right) \in D_{1}$. Имеем

$$
\left|\sum_{s=0}^{n-1} f_{s}(k(t)) \frac{u(k(t))^{s}}{v(k(t))^{s}}-\frac{\left[u(k(t))-\overline{k\left(w_{0}\right)} v(k(t))\right]^{n-1}}{v(k(t))^{n-1}\left[k(t)-k\left(w_{0}\right)\right]}\right|<\delta
$$


для п.в. $t \in \Gamma_{1}$, в которых выполняется $(2.1)$ (в частности, $k(t)$ определено и принадлежит $\partial \Omega$ ). То есть

$$
\begin{gathered}
\left|\sum_{s=0}^{n-1} f_{s}(k(t)) u(k(t))^{s} v(k(t))^{n-1-s}\left[k(t)-k\left(w_{0}\right)\right]-\left[u(k(t))-\overline{k\left(w_{0}\right)} v(k(t))\right]^{n-1}\right| \\
\leqslant \delta \sup _{\tau \in \Gamma_{1}}\left|v(k(\tau))^{n-1}\left[k(\tau)-k\left(w_{0}\right)\right]\right|
\end{gathered}
$$

для п.в. $t \in \Gamma$. Заметим, что функции в левой и правой частях неравенства (2.2) (знаки модулей опускаются) представляют собой граничные значения соответствующих функций из $H_{\infty}\left(D_{1}\right)$, поэтому по принципу максимума модуля мы можем подставить $w_{0}$ вместо $t$ в $(2.2)$ :

$$
\left|\left[u\left(k\left(w_{0}\right)\right)-\overline{k\left(w_{0}\right)} v\left(k\left(w_{0}\right)\right)\right]^{n-1}\right| \leqslant \delta \sup _{\tau \in \Gamma_{1}}|v(k(\tau))|^{n-1}\left|k(\tau)-k\left(w_{0}\right)\right|
$$

что дает противоречие при достаточно малых $\delta$.

Обратно, пусть $C(\partial \Omega) \neq \mathscr{R}_{n}(\partial \Omega, \bar{\Omega})$, тогда $C(\partial \Omega) \neq \mathscr{R}_{2}(\partial \Omega, \bar{\Omega})$. Поэтому найдется мера $\mu \not \equiv 0$ на $\partial \Omega$ такая, что $\mu \perp \mathscr{R}_{1}(\partial \Omega, \bar{\Omega})$ и $\bar{\zeta} \mu \perp \mathscr{R}_{1}(\partial \Omega, \bar{\Omega})$ (символ $\perp$ означает ортогональность). Так как $\Omega$ является областью Каратеодори, то согласно [7; лемма 9] меры $\mu$ и $\bar{\zeta} \mu$ не имеют атомов.

Пусть $F(z)=\widehat{\mu}(z), G(z)=\widehat{\bar{\zeta}} \mu(z), f(w)=F(k(w)) k^{\prime}(w)$ и $g(w):=G(k(w)) k^{\prime}(w)$. Мы утверждаем, что $f, g \in H_{1}\left(D_{1}\right)$. Нам будет удобно сформулировать это (скорее всего, известное) утверждение в виде леммы и привести его доказательство.

Лемма 2.3. Пусть $\Omega$ - область Каратеодори в $\mathbb{C}, \nu-$ мера на $\partial \Omega$, ортогональная $\kappa \mathscr{R}_{1}(\partial \Omega, \bar{\Omega})$, a $k$ - конформное отображсение $D_{1}$ на $\Omega$. Тогда $\widehat{\nu}(k(w)) k^{\prime}(w) \in H_{1}\left(D_{1}\right)$.

ДокАЗАТЕЛЬСТво. Пусть $z_{0}=k(0)$. Без ограничения общности будем считать (в этом доказательстве), что $k^{\prime}(0)>0$. Выберем последовательность спрямляемых контуров $\left\{T_{m}\right\}_{m=1}^{\infty}$ таких, что $\Omega \subset D\left(T_{m}\right) \subset D\left(T_{m-1}\right)$ и $\left\{T_{m}\right\}$ сходится к $\partial \Omega$ при $m \rightarrow \infty$ (в качестве $T_{m}$ может быть взят образ окружности $\{|w|=1-1 /(m+1)\}$ при каком-либо фиксированном конформном отображении круга $\{|w|<1\}$ на $\left.\Omega_{\infty}\right)$. Пусть $\varphi_{m}: D\left(T_{m}\right) \rightarrow D_{1}-$ конформньй изоморфизм с нормализацией $\varphi_{m}\left(z_{0}\right)=0, \varphi_{m}^{\prime}\left(z_{0}\right)>0$. По теореме Каратеодори [8; c. 74-80] последовательность $\left\{\varphi_{m}\right\}$ сходится к $k^{-1}$ равномерно на компактах из $\Omega$. Зафиксируем произвольную точку $w \in D_{1}$ и положим $z_{m}=\varphi_{m}^{-1}(w)$. Функция

$$
\left\{\begin{array}{l}
h(\zeta)=\frac{1}{\varphi_{m}(\zeta)-\varphi_{m}\left(z_{m}\right)}-\frac{1}{\varphi_{m}^{\prime}\left(z_{m}\right)\left(\zeta-z_{m}\right)} \text { при } \zeta \neq z_{m} \\
h\left(z_{m}\right)=-\frac{\varphi_{m}^{\prime \prime}\left(z_{m}\right)}{2 \varphi_{m}^{\prime}\left(z_{m}\right)}
\end{array}\right.
$$

голоморфна в $D\left(T_{m}\right)$ (т.е. $\left.h \in \mathscr{R}_{1}(\partial \Omega, \bar{\Omega})\right)$, а $\nu \perp \mathscr{R}_{1}(\partial \Omega, \bar{\Omega})$, поэтому

$$
\frac{1}{2 \pi i} \int \frac{d \nu(\zeta)}{\varphi_{m}(\zeta)-\varphi_{m}\left(z_{m}\right)}=\frac{\widehat{\nu}\left(z_{m}\right)}{\varphi_{m}^{\prime}\left(z_{m}\right)}
$$


Определим меры $\nu_{m}$ по формулам $\nu_{m}(S)=\nu\left(\varphi_{m}^{-1}\left(S \cap D_{1}\right)\right)$ для произвольного борелевского множества $S \subset \mathbb{C}$. Тогда $\operatorname{Spt} \nu_{m} \subset D_{1}$ и имеет место равенство

$$
\frac{1}{2 \pi i} \int \frac{d \nu_{m}(\tau)}{\tau-w}=\widehat{\nu}\left(\varphi_{m}^{-1}(w)\right)\left(\varphi_{m}^{-1}\right)^{\prime}(w)
$$

причем $\nu_{m} \perp \mathscr{P}_{1}$ и $\left\|\nu_{m}\right\| \leqslant\|\nu\|$. Пусть $\nu_{0}$ - какая-либо предельная точка последовательности $\left\{\nu_{m}\right\}$ в $*$-слабой топологии (пространства мер). Тогда Spt $\nu_{0} \subset \Gamma_{1}$ и $\nu_{0} \perp \mathscr{P}_{1}\left(\Gamma_{1}\right)$.

По известной теореме $\Phi$. и М. Риссов (см., например, [5; гл. $2, \S 5])$ найдется функция $h \in H_{1}\left(D_{1}\right)$ (угловые граничные значения которой мы обозначим через $h(t))$ с условием $\nu_{0}=h(t) d t_{\mid \Gamma_{1}}$. Переходя к пределу в $(2.3)$, получаем

$$
\widehat{\nu_{0}}(w)=\widehat{\nu}(k(w)) k^{\prime}(w)=\frac{1}{2 \pi i} \int_{\Gamma_{1}} \frac{d \nu_{0}(t)}{t-w}=\frac{1}{2 \pi i} \int_{\Gamma_{1}} \frac{h(t) d t}{t-w}=h(w)
$$

для всех $w \in D_{1}$, так что $\widehat{\nu}(k(w)) k^{\prime}(w) \in H_{1}\left(D_{1}\right)$ и лемма доказана.

Положим

$$
\Psi(z)=\bar{z} F(z)-G(z)=\frac{1}{2 \pi i} \int \frac{\bar{z}-\bar{\zeta}}{\zeta-z} d \mu(\zeta)
$$

Так как ядро $\frac{\bar{z}-\bar{\zeta}}{\zeta-z}$ ограничено и $\mu$ не имеет атомов, то $\Psi \in C(\mathbb{C})$. Кроме того, при фиксированном $z \in \mathbb{C} \backslash \bar{\Omega}$ имеем $\frac{\bar{z}-\bar{\zeta}}{\zeta-z} \in \mathscr{R}_{2}(\partial \Omega, \bar{\Omega})$, т.е. $\Psi(z)=0$ при $z \in \mathbb{C} \backslash \bar{\Omega}$. Следовательно, $(\bar{z} F(z)-G(z)) \rightarrow 0$ при $z \rightarrow \partial \Omega, z \in \Omega$. Отсюда вытекает, что

$$
\frac{f(w) \overline{k(w)}-g(w)}{k^{\prime}(w)}=F(k(w)) \overline{k(w)}-G(k(w))=\bar{z} F(z)-G(z) \rightarrow 0
$$

при $w \rightarrow \Gamma_{1}, w \in D_{1}$ (здесь $\left.z=k(w)\right)$.

Так как $f, g \in H_{1}\left(D_{1}\right)$, то функция $f(w) \overline{k(w)}-g(w)$ имеет п.в. на $\Gamma_{1}$ угловые предельные значения. Кроме того, для п.в. $t \in \Gamma_{1}$ и для любого угла Штольца $\Theta_{t}$ с вершиной в точке $t$ (т.е. $\Theta_{t}$ - это угол с раствором $\alpha<\pi$ с вершиной в точке $t$, биссектриса которого проходит через центр круга $\left.D_{1}\right)$ сушествует последовательность $\left\{w_{l}\right\} \in \Theta_{t}$ такая, что $w_{l} \rightarrow t$ при $l \rightarrow \infty$ и $\limsup _{l \rightarrow \infty}\left|k^{\prime}\left(w_{l}\right)\right|<\infty$. В самом деле, если это не так, то функция $1 / k^{\prime}(w)$ имеет нулевые угловые значения на множестве положительной длины на $\Gamma_{1}$, что невозможно в силу граничной теоремы единственности Лузина-Привалова.

Итак, для п.в. $t \in \Gamma_{1}$ имеет место $f(t) \overline{k(t)}-g(t)=0$. Заметим, что $f \not \equiv 0$, так как $\mu \not \equiv 0$ (см. [7; лемма 4]). Поскольку $H_{1}\left(D_{1}\right) \subset N\left(D_{1}\right)$ (класс Неванлинны) и (согласно [5; гл. 2, раздел 2.1]) всякая функция класса Неванлинны представляется в виде отношения двух функций класса $H_{\infty}\left(D_{1}\right)$, то для п.в. $t \in \Gamma_{1}$ имеем

$$
\overline{k(t)}=\frac{g(t)}{f(t)}=\frac{g_{1}(t)}{f_{1}(t)}=\frac{u(k(t))}{v(k(t))}
$$

где $f_{1}, g_{1} \in H_{\infty}\left(D_{1}\right), u, v \in H_{\infty}(\Omega)$. Таким образом, $\Omega \in \mathscr{N}$ и утверждение (1) теоремы 2.2 полностью доказано. 
Докажем утверждение (2). Пусть найдется ограниченная компонента $\Omega$ в $\mathbb{C} \backslash X$ с условием $\Omega \in \mathscr{N}$. Тогда согласно утверждению (1) теоремы 2.2 существует ненулевая мера $\mu$ с носителем Spt $\mu \subset \partial \Omega$ такая, что $\mu \perp \mathscr{R}_{n}(\partial \Omega, \bar{\Omega})$ и, следовательно, $\mu \perp \mathscr{P}_{n}(X)$. Так как $\mu \not \equiv 0$ и $\widehat{\mu}(z)=0$ вне $\bar{\Omega}$, то в силу [7; лемма 4] существует такая точка $z_{0} \in \Omega$, что $\widehat{\mu}\left(z_{0}\right) \neq 0$. Это означает, что $\mu \not \perp\left(z-z_{0}\right)^{-1} \in \mathscr{A}_{n}(X)$, т.е. $\mathscr{A}_{n}(X) \neq \mathscr{P}_{n}(X)$ и первая часть утверждения (2) проверена.

Для доказательства обратного утверждения в (2) нам потребуется лемма, являющаяся непосредственным следствием [7; лемма 7] и известной теоремы Рунге:

ЛЕмма 2.4. Пусть $X$ - компакт Каратеодори, $\Omega$ - некоторая ограниченная компонента его дополнения, $U=(\widehat{X})^{\circ} \backslash \Omega$. Тогда существует последовательность $\left\{q_{j}\right\} \subset \mathscr{P}_{1}$ с условиями:

1) $q_{j} \rightrightarrows 1$ на каждом компакте в $\Omega$;

2) $q_{j} \rightrightarrows 0$ накаждом компакте в $U$;

3) $\left\|q_{j}\right\|_{\widehat{X}} \leqslant C$, где $C$ - абсолютная константа.

Пусть $\mu$ - произвольная ненулевая мера на $X, \mu \perp \mathscr{P}_{n}(X)$. Фиксируем произвольную ограниченную компоненту $\Omega$ множества $\mathbb{C} \backslash X$. По условию $\Omega \notin \mathscr{N}$. Мы утверждаем, что $\widehat{\bar{\zeta}^{s}} \mu(z)=0$ при $z \in \Omega$ и $s=0, \ldots, n-1$. Действительно, выберем согласно лемме 2.4 последовательность $\left\{q_{j}\right\} \subset \mathscr{P}_{1}$ для рассматриваемых $X, \Omega$ и $U=(\widehat{X})^{\circ} \backslash \Omega$. Определим меры $\mu_{j}=q_{j} \mu$. Пусть $\mu_{\Omega}-$ некоторая предельная точка последовательности $\left\{\mu_{j}\right\}$ в $*$-слабой топологии пространства мер (т.е. сушествует последовательность $\left\{j_{l}\right\}$ такая, что $j_{l} \rightarrow+\infty$ и $\mu_{j_{l}} \stackrel{*}{\rightarrow} \mu_{\Omega}$ при $\left.l \rightarrow+\infty\right)$. Ясно, что Spt $\mu_{\Omega} \subset \partial X$ и для любых $s=0, \ldots, n-1$ и $z_{0} \notin \operatorname{Spt} \mu$ имеем

$$
\begin{aligned}
\widehat{\bar{\zeta}^{s} \mu_{\Omega}}\left(z_{0}\right) & =\frac{1}{2 \pi i} \int \frac{\bar{z}^{s} d \mu_{\Omega}(z)}{z-z_{0}}=\lim _{l \rightarrow \infty} \frac{1}{2 \pi i} \int \frac{\bar{z}^{s} q_{j_{l}}(z) d \mu(z)}{z-z_{0}} \\
& =\lim _{l \rightarrow \infty}\left(\frac{1}{2 \pi i} \int \frac{\left(q_{j_{l}}(z)-q_{j_{l}}\left(z_{0}\right)\right) \bar{z}^{s} d \mu(z)}{z-z_{0}}+\frac{q_{j_{l}}\left(z_{0}\right)}{2 \pi i} \int \frac{\bar{z}^{s} d \mu(z)}{z-z_{0}}\right) \\
& =\lim _{l \rightarrow \infty} \frac{q_{j_{l}}\left(z_{0}\right)}{2 \pi i} \int \frac{\bar{z}^{s} d \mu(z)}{z-z_{0}}= \begin{cases}\widehat{\bar{\zeta}^{s}} \mu\left(z_{0}\right) & \text { при } z_{0} \in \Omega, \\
0 & \text { при } z_{0} \in(U \backslash \operatorname{Spt} \mu) \cup \Omega_{\infty}^{\prime},\end{cases}
\end{aligned}
$$

где $\Omega_{\infty}^{\prime}$ - неограниченная компонента $\mathbb{C} \backslash X$. Из последнего вытекает, что $\mu_{\Omega} \perp$ $\mathscr{P}_{n}(X)$, и мы (для доказательства того, что $\widehat{\bar{\zeta}^{s}} \mu(z)=0$ на $\left.\Omega\right)$ можем $(u$ будем $)$ считать, что Spt $\mu \subset \partial X$ (достаточно рассмотреть вместо $\mu$ меру $\mu_{\Omega}$ и повторить рассуждение). Но тогда из предыдущей серии равенств мы получаем, что $\widehat{\bar{\zeta}^{s} \mu_{\Omega}}\left(z_{0}\right)=0$ на $U \cup \Omega_{\infty}^{\prime}$. Поскольку Spt $\mu \subset \partial X$ и $\mu \perp \mathscr{P}_{1}(X)$, то из [7; лемма 9$]$ следует, что $\mu$ (и, следовательно, $\mu_{\Omega}$ ) не имеет атомов. Функция

$$
f_{\Omega}(w)=\frac{1}{\pi} \int \frac{(\bar{z}-\bar{w}) d \mu_{\Omega}(z)}{z-w}
$$

непрерывна на $\mathbb{C}$ и обрашается в нуль на $U \cup \Omega_{\infty}^{\prime}$, так что $f_{\Omega}(w)=0$ вне $\bar{\Omega}$. Как известно, $\bar{\partial}^{2} f_{\Omega}=\mu_{\Omega}$ в обобщенном смысле, так что Spt $\mu_{\Omega} \subset \partial \Omega$ и, следовательно, $\mu_{\Omega} \perp \mathscr{R}_{n}(\partial \Omega, \bar{\Omega})$. Так как $\Omega \notin \mathscr{N}$, то согласно утверждению (1) теоремы $\mu_{\Omega}=0$ и нужное утверждение получено. Таким образом, доказано, что для любой компоненты $\Omega$ дополнения $\mathbb{C} \backslash X$ имеет место $\widehat{\bar{\zeta}^{s}} \mu(z)=0$ при $z \in \Omega$ и $s=0, \ldots, n-1$, т.е. $\mu \perp \mathscr{R}_{n}(X, X)$.

Для доказательства теоремы 2.2 остается установить следующее 
ПРЕДЛОЖЕНИЕ 2.5. Если $X$ - компакт Kаратеодори, то $\mathscr{R}_{n}(X, X)=$ $\mathscr{A}_{n}(X)$ при всех натуральных $n$.

ДокАЗАтЕльство. Случай $n=1$ непосредственно вытекает из емкостного критерия Витушкина [9; гл. 2, §5, теорема 1], а случай $n=2$ - из [10; теорема 3.1$]$. При $n>2$ доказательство проводится по той же схеме (схеме Витушкина) или аналогично доказательству в [3]. Тем не менее для удобства читателя мы обсудим основные моменты доказательства ввиду его технической сложности.

Положим $L=\bar{\partial}^{n}$, и пусть $\Phi(z)=\bar{z}^{n-1}((n-1) ! \pi z)^{-1}$ - фундаментальное решение для $L, \partial:=\partial / \partial z, B(a, r)$ - открытый круг с центром $a$ и радиусом $r>0$. Зафиксируем какую-либо $f \in \mathscr{A}_{n}(X)$ и продолжим ее до непрерьвной функции в $\mathbb{C}$ с компактньм носителем.

Теперь зафиксируем произвольное $\delta>0$ и построим стандартное $\delta$-разбиение единицы $\left\{\varphi_{j}\right\}_{j \in \mathbb{Z}^{2}}$ на $\mathbb{C}$, где $\varphi_{j} \in C_{0}^{\infty}\left(B\left(a_{j}, \delta\right)\right)$ и $a_{j}=\delta j$ (см. [10]). Пусть $J=$ $\left\{j \in \mathbb{Z}^{2}: B\left(a_{j}, \delta\right) \cap \partial X \neq \varnothing\right\}$. При каждом $j \in J$ найдется $a_{j}^{*} \in B\left(a_{j}, \delta\right) \backslash X$ и жорданова кривая $\gamma_{j}$ в $\overline{B\left(a_{j}^{*}, \delta\right)} \backslash X$ с началом в точке $a_{j}^{*}$ и концом на $\partial B\left(a_{j}^{*}, \delta\right)$. При $j \notin J$ положим $a_{j}^{*}=a_{j}$.

Пусть $f_{j}=\Phi *\left(\varphi_{j} L f\right)$, так что $L f_{j}=\varphi_{j} L f$ в обобщенном смысле и

$$
f_{j}(z)=\sum_{s=0}^{n-1} \sum_{m=0}^{\infty} c_{m}^{s} \bar{\partial}^{s} \partial^{m} \Phi\left(z-a_{j}^{*}\right),
$$

где ряд (типа Лорана) сходится в метрике $C^{\infty}\left(\left|z-a_{j}^{*}\right|>2 \delta\right)\left(c_{m}^{s}\right.$ зависят от $\left.j\right)$. Стандартным образом доказывается (см. [10]), что

$$
\begin{gathered}
\left\|f_{j}\right\|_{B\left(a_{j}^{*}, 3 \delta\right)} \leqslant A \omega(\delta), \\
\left|c_{m}^{s}\right| \leqslant \frac{A \omega(\delta)(2 \delta)^{2-n+m+s}}{m ! s !}, \quad s=0,1, \ldots, n-1, \quad m=0,1, \ldots .
\end{gathered}
$$

Здесь и далее (в этом доказательстве) константа $A$ зависит только от $n$ и может меняться в разных соотношениях.

Установим аналог леммы 3.1 из [10]. Для произвольно взятой жордановой кривой $\Gamma$ в $\overline{B(0, \delta)}$ с началом в точке 0 и концом $a,|a|=\delta$, построим функцию $g_{\Gamma}$ следуюшим образом. Пусть

$$
h(z)=c_{0}\left(z^{n}\left(z-\delta^{-1} a\right)^{n} \sqrt{z\left(z-\delta^{-1} a\right)}-p_{0}(z)\right),
$$

где голоморфная ветвь корня (вне кривой $\delta^{-1} \Gamma$ ), константа $c_{0}$ и полином $p_{0}$ (не зависящий от $\left.\delta^{-1} \Gamma\right)$ подобраны так, что $\lim _{z \rightarrow \infty} z h(z)=1$. Теперь полагаем

$$
g_{\Gamma}(z)=\frac{\bar{z}^{n-1} h(z / \delta)}{(n-1) ! \pi \delta}
$$

так что

$$
\left\|\bar{\partial}^{s} \partial^{m} g_{\Gamma}\right\|_{B(0,3 \delta) \backslash \Gamma} \leqslant A \delta^{n-2-s-m}, \quad s=0,1, \ldots, n-1, \quad m=0,1, \ldots, n .
$$

Кроме того,

$$
g_{\Gamma}(z)=\sum_{m=0}^{\infty} d_{m} \partial^{m} \Phi(z)
$$


при $|z|>\delta$, причем $d_{0}=1$ и справедливы оценки:

$$
\left|d_{m}\right| \leqslant \frac{A \delta^{m}}{m !}, \quad m=0,1, \ldots
$$

Следующие неравенства элементарны:

$$
\left|\bar{\partial}^{s} \partial^{m} \Phi(z)\right| \leqslant A m !|z|^{n-2-s-m}, \quad s=0,1, \ldots, n-1, \quad m=0,1, \ldots .
$$

При $j \in J$ положим $\gamma_{j}^{*}=\gamma_{j}-a_{j}^{*}$, так что определены $g_{\gamma_{j}^{*}}\left(z-a_{j}^{*}\right)$ и мы можем ввести функции

$$
g_{j}(z):=\sum_{s=0}^{n-1} \sum_{l=0}^{n-s} \beta_{l}^{s} \bar{\partial}^{s} \partial^{l} g_{\gamma_{j}^{*}}\left(z-a_{j}^{*}\right)=: \sum_{s=0}^{n-1} \sum_{m=0}^{\infty} b_{m}^{s} \bar{\partial}^{s} \partial^{m} \Phi\left(z-a_{j}^{*}\right)
$$

(последний ряд сходится в $\left.C^{\infty}\left(\left\{\left|z-a_{j}^{*}\right|>2 \delta\right\}\right)\right)$, где $n(n+3) / 2$ коэффициентов $\beta_{l}^{s}$ могут быть найдены из следующих условий:

$$
c_{m}^{s}=b_{m}^{s}, \quad s=0, \ldots, n-1, \quad m=0, \ldots, n-s .
$$

Пусть

$$
g_{\gamma_{j}^{*}}\left(z-a_{j}^{*}\right)=\sum_{m=0}^{\infty} d_{m}^{*} \partial^{m} \Phi\left(z-a_{j}^{*}\right),
$$

где $d_{0}^{*}=1$ и $\left|d_{m}^{*}\right| \leqslant A \delta^{m} / m !, m=0,1, \ldots$ Тогда

$$
b_{m}^{s}=\sum_{l=0}^{\min \{m, n-s\}} \beta_{l}^{s} d_{m-l}^{*}, \quad s=0, \ldots, n-1, \quad m=0,1, \ldots
$$

Мы получаем следующую (распадающуюся по $s$ треугольную) систему уравнений на $\beta_{l}^{s}$ :

$$
c_{m}^{s}=\sum_{l=0}^{m} \beta_{l}^{s} d_{m-l}^{*}, \quad s=0, \ldots, n-1, \quad m=0, \ldots, n-s .
$$

Отсюда получаем $\left|\beta_{l}^{s}\right| \leqslant A \omega(\delta) \delta^{2-n+l+s}$ и, следовательно,

$$
\begin{gathered}
\left\|g_{j}\right\|_{B\left(a_{j}^{*}, 3 \delta\right)} \leqslant A \omega(\delta), \\
\left|b_{m}^{s}\right| \leqslant \frac{A \omega(\delta)(2 d)^{2-n+m+s} m^{n}}{m ! s !}, \quad s=0,1, \ldots, n-1, \quad m=0,1, \ldots .
\end{gathered}
$$

Таким образом,

$$
\left|f_{j}(z)-g_{j}(z)\right| \leqslant A \omega(\delta) \min \left(1, \frac{\delta^{3}}{\left|z-a_{j}^{*}\right|^{3}}\right)
$$

для всех $z \in \mathbb{C}$ (вне $B\left(a_{j}^{*}, 3 \delta\right)$ используются приведенные выше оценки для $c_{m}^{s}$, $b_{m}^{s}, \bar{\partial}^{s} \partial^{m} \Phi\left(z-a_{j}^{*}\right)$ и соответствующие разложения типа Лорана с центром $\left.a_{j}^{*}\right)$. Наконец, в последнем неравенстве $a_{j}^{*}$ можно заменить на $a_{j}$. Из леммы Витушкина 
$[9 ;$ гл. $2, \S 4$, лемма 1$]$ следует, что функция $f$ равномерно на $X$ приближается функциями $\sum_{j \in J} g_{j}+\sum_{j \notin J} f_{j}$ при $\delta \rightarrow 0$. Поскольку $L g_{j}=0$ в окрестности $X, f_{j} \equiv 0$ при $B\left(a_{j}, \delta\right) \subset X^{\circ}$ и $L f_{j}=0$ вне $B\left(a_{j}, \delta\right)$, остается воспользоваться классической теоремой Рунге.

Вопрос о нахождении условий на компакт $X$, необходимых и достаточных для вьполнения равенства $\mathscr{R}_{n}(X, X)=\mathscr{A}_{n}(X)$, представляет самостоятельный интеpec. Отметим работы [11]-[13], связанные с этой задачей. Так, в работах [3; §4] и [13] поставлена следуюшая задача, которая пока остается нерешенной: верно ли, что для любого компакта $X$ в $\mathbb{C}$ имеет место равенство $\mathscr{A}_{2}(X)=\mathscr{R}_{2}(X, X)$ ?

Заметим, что если $\mathbb{C} \backslash \bar{\Omega}$ связно, то по теореме Рунге можно показать, что $\mathscr{R}_{n}(\partial \Omega, \bar{\Omega})=\mathscr{P}_{n}(\partial \Omega)$. Из этого факта и из теоремы $2.2(1)$ вытекает

СлЕДСТвИЕ 2.6. Если $\Omega$ - область Каратеодори и $\mathbb{C} \backslash \bar{\Omega}$ связно, а $n \geqslant 2$ натуральное число, то $C(\partial \Omega)=\mathscr{P}_{n}(\partial \Omega)$, если и только если $\Omega \notin \mathscr{N}$.

\section{§3. Свойства неванлинновских областей. Примеры наличия и отсутствия аппроксимации}

Оказывается, что свойство $\Omega \in \mathscr{N}$ имеет следующую характеристику в терминах конформных отображений $D_{1}$ на $\Omega$.

ПРЕДЛОЖЕНИЕ 3.1. Пусть $\Omega$ - ограниченная односвязная область в $\mathbb{C}$. Свойство $\Omega \in \mathscr{N}$ имеет место тогда и только тогда, когда конформное отображение $k$ единичного круга $D_{1}$ на область $\Omega$ допускает псевдопродолжение неванлинновского типа через $\Gamma_{1}$ на $\overline{\mathbb{C}} \backslash \overline{D_{1}}$. Это означает, что существуют такие ограниченные голоморфнье в $\overline{\mathbb{C}} \backslash \overline{D_{1}}$ функции $u_{1}(\cdot)$ и $v_{1}(\cdot)$ $\left(v_{1} \not \equiv 0\right)$, что при $\widetilde{k}(w)=u_{1}(w) / v_{1}(w),|w|>1$, для n.в. $t \in \Gamma_{1}$ имеет место равенство $\widetilde{k}(t)=k(t)$ (здесь $k(t)$ суть угловье граничнье значения $k$ изнутри $D_{1}, a \widetilde{k}(t)$ - угловые граничные значения $\widetilde{k}$ изнутри $\left.\mathbb{C} \backslash \overline{D_{1}}\right)$.

ДокАЗАТЕльСтво. Для произвольной функции $g(\cdot)$ положим $g_{*}(w)=\overline{g(\bar{w})}$ для всех $w$, где $g_{*}$ определена.

Пусть $\Omega \in \mathcal{N}$, и пусть $u$ и $v$ взяты из определения 2.1. При $|w|>1$ определим $u_{1}(w)=(u(k(1 / w)))_{*}$ и $v_{1}(w)=(v(k(1 / w)))_{*}$. В силу $(2.1)$ для п.в. $t \in \Gamma_{1}$ имеем, что если $w \in \mathbb{C} \backslash \overline{D_{1}}$ некасательно стремится к $t$, то

$$
\widetilde{k}(w)=\frac{u_{1}(w)}{v_{1}(w)}=\frac{(u(k(1 / w)))_{*}}{(v(k(1 / w)))_{*}}=\frac{\overline{u\left(k\left(w^{\prime}\right)\right)}}{\overline{v\left(k\left(w^{\prime}\right)\right)}} \rightarrow k(t),
$$

так как $w^{\prime}=1 / \bar{w} \in D_{1}$ также некасательно стремится к $t$.

Обратно, пусть существуют функции $u_{1}$ и $v_{1}$ и вьполнено условие псевдопродолжения. Определим при $|w|<1$ функции $U(w)=\left(u_{1}(1 / w)\right)_{*}$ и $V(w)=\left(v_{1}(1 / w)\right)_{*}$. Как и в предыдушем случае, для п.в. $t \in \Gamma_{1}$ получаем, что если $w \in D_{1}$ некасательно стремится к $t$, то

$$
\frac{U(w)}{V(w)}=\frac{\left(u_{1}(1 / w)\right)_{*}}{\left(v_{1}(1 / w)\right)_{*}}=\frac{\overline{u_{1}\left(w^{\prime}\right)}}{\overline{v_{1}\left(w^{\prime}\right)}} \rightarrow \overline{\widetilde{k}(t)}=\overline{k(t)},
$$

так как $w^{\prime}=1 / \bar{w} \in \mathbb{C} \backslash \overline{D_{1}}$ некасательно стремится к $t$. Остается положить $u(z)=U\left(k^{-1}(z)\right)$ и $v(z)=V\left(k^{-1}(z)\right)$. 
Применим предложение 3.1 к случаю жордановых неванлинновских областей, границы которых будем называть неванлинновскими контурами. Совокупность всех неванлинновских контуров мы также будем обозначать через $\mathscr{N}$. Понятие неванлинновского контура (в несколько других терминах) было введено в [4] (для спрямляемых контуров) и в [14] (для произвольного контура). Утверждение следствия 2.6 для жордановых областей было получено в [14].

Заметим, что для области $\Omega=D(\Gamma)$, ограниченной спрямляемьм контуром $\Gamma$, свойство $\bar{\zeta}=u(\zeta) / v(\zeta)$ может пониматься напрямую - как равенство соответствующих угловых граничных значений п.в. на $\Gamma$.

В частном случае, когда конформное отображение $k$ круга $D_{1}$ на область $D(\Gamma)$ может быть продолжено до отображения, конформного в некоторой окрестности множества $\overline{D_{1}}$ (это в точности означает, что $\Gamma$ - аналитический контур), нетрудно показать, что функция

$$
S(z)=k_{*}\left(\frac{1}{k^{-1}(z)}\right)
$$

голоморфна и однолистна в некоторой связной окрестности $U$ контура Г. Эту окрестность можно выбрать так, чтобы

$$
\Gamma=\{\zeta \in U: \bar{\zeta}=S(\zeta)\}
$$

Функция $S$ называется функиией ШІварца контура $Г$. Очевидно, что $S$ однозначно определена в некоторой связной окрестности контура Г (см. [15], где подробно изучаются свойства этой функции). В случае, когда $Г$ - аналитический контур, из предложения 3.1 вытекает, что $\Gamma \in \mathscr{N}$ тогда и только тогда, когда $k$ является рациональной функцией с полюсами вне $\overline{D_{1}}$ (см. также [15; гл. 14]). В последнем случае $S=u / v$ имеет конечное число особых точек (полюсов) в $D(\Gamma)$.

Рассмотрим ряд конкретных примеров. Очевидно, $\Gamma_{1}=\{t \in \mathbb{C}: \bar{t}=1 / t\} \in \mathscr{N}$. Функцию Шварца для эллипса

$$
\Gamma_{a b}=\left\{z=x+i y: \frac{x^{2}}{a^{2}}+\frac{y^{2}}{b^{2}}=1\right\}, \quad 0<b<a,
$$

легко вычислить непосредственно:

$$
S(z)=\frac{\left(a^{2}+b^{2}\right) z-2 a b \sqrt{z^{2}-c^{2}}}{c^{2}} .
$$

Здесь $c=\sqrt{a^{2}-b^{2}}>0$ и в (3.3) берется та голоморфная ветвь $s(z)$ многозначной функции $\sqrt{z^{2}-c^{2}}$ вне $[-c, c]$, которая удовлетворяет условию $s(a)=b$. Таким образом, $\Gamma_{a b} \notin \mathscr{N}$.

Пусть теперь $\Omega$ - односвязная ограниченная область с гранищей, содержащей две (достижимые из $\Omega$ ) аналитические дуги, причем функции Шварца этих дуг (в соответствующих окрестностях этих дуг) задают аналитические элементы, не продолжаемые друг в друга (такие дуги назовем аналитически независимыми). Из теоремы единственности Лузина-Привалова вытекает, что в этом случае $\Omega \notin \mathscr{N}$ (см. также [4; предложение 2]). Таковьми являются все многоугольники.

Проиллюстрируем применение теоремы 2.2. Пусть $\Omega$ - "рог изобилия", представляющий собой область, ограниченную двумя спиралями $z=\left(1+e^{-\theta}\right) e^{i \theta}$ и $z=\left(1+2 e^{-\theta}\right) e^{i \theta}, \theta \in[0,+\infty)$, а также отрезком $[2,3]$ вешественной оси. Так 
как $\partial \Omega$ содержит аналитически независимые дуги (достижимые из $\Omega$ ), то $\Omega \notin \mathscr{N}$. Заметим, что $\Gamma_{1} \subset \partial \Omega$. По теореме $2.2(1) C(\partial \Omega)=\mathscr{R}_{n}(\partial \Omega, \bar{\Omega}) \neq \mathscr{P}_{n}(\partial \Omega)$ при любом $n \geqslant 2$. С другой стороны, по теореме $2.2(2)$ для $X=\partial \Omega \cup D_{1}$ имеет место $\mathscr{A}_{n}(X)=\mathscr{P}_{n}(X)$ для любого $n \geqslant 2$. Пусть теперь $\Omega_{1}$ является образом области $\Omega$ под действием преобразования $(x+i y) \rightarrow(x+i y / \sqrt{2})$. По теореме $2.2(2)$ "рог изобилия" $\Omega_{1}$, "накручивающийся" на эллипс $\left\{x^{2}+2 y^{2}=1\right\}$, обладает тем свойством, что $C\left(\partial \Omega_{1}\right)=\mathscr{P}_{n}\left(\partial \Omega_{1}\right)$ при $n \geqslant 2$.

Другие важные примеры неванлинновских областей можно найти в [16] и [17; п. 4.2, пример 5].

Изучение геометрических свойств неванлинновских областей и контуров представляет особый интерес. Естественно возникает, например, следующая задача.

ЗАДАчА 3.2. Верно ли, что всякий неванлинновский контур спрямляем?

ПРЕДЛОЖЕНИЕ 3.3. Если D - жорданова область со спрямляемой границей $\Gamma$ и $D \in \mathcal{N}$, то функиия $u-\bar{z} v$ (для всех допустимых и и $v$, см. определение 2.1) непрерывно продолжается из области $D$ на $\bar{D}$, причем $u-\bar{z} v=0$ на Г.

Доказательство этого предложения приведено в $\S 5$ сразу после доказательства утверждения (1) теоремы 5.5.

В завершение 55 будет построен спрямляемый неванлинновский контур Г такой, что соответствуюшие функции $u(\cdot)$ и $v(\cdot)$ не могут быть выбраны (ни одна из них) из пространства $\mathscr{A}_{1}(\overline{D(\Gamma)})$ (см. пример 5.8).

П. Ахерн обратил наше внимание на следующий результат Р. Дугласа, Г. Шапиро и А. Шилдса [18] (который мы сформулируем в нашей терминологии): ограниченная односвязная область $\Omega$ является неванлинновской тогда и только тогда, когда $k$ не является циклическим вектором оператора обратного сдвига $f \mapsto(f(z)-f(0)) / z$ в пространстве $H_{2}\left(D_{1}\right)$ (см. [18; теорема 2.2.1]).

\section{§4. Аппроксимация на объединении компактных множеств}

Фиксируем натуральное $n \geqslant 2$. Исследуемьй в этом параграфе вопрос может быть кратко сформулирован следующим образом. Пусть $X_{1}$ u $X_{2}-$ компакть в $\mathbb{C}, X=X_{1} \cup X_{2} ;$ когда для $X$ справедливо равенство (1.2)?

Заметим, что в общей ситуации указанньй вопрос не вполне удачен: возможно все. Например, оба компакта $X_{1}=\{z:|z|=1, \operatorname{Re} z \leqslant 0\}$ и $X_{2}=\{z:|z|=1$, $\operatorname{Re} z \geqslant 0\}$ удовлетворяют (1.2), что не так для $X=X_{1} \cup X_{2}$. Противоположная ситуация возникает в случае $X_{1}=\{z:|z-1|=1\} \cup\{z:|z+1| \leqslant 1\}$ и $X_{2}=\{z$ : $|z+1|=1\} \cup\{z:|z-1| \leqslant 1\}$, достаточно воспользоваться теоремами 1.3 и 2.2 . $\mathrm{C}$ другой стороны, если $X_{1} \cap X_{2}=\varnothing$, то (1.2) может иметь место при $X=X_{1} \cup X_{2}$, только если (1.2) справедливо для каждого из $X_{1}$ и $X_{2}$ по отдельности. Более того, последнее условие также и достаточно, если $\widehat{X_{1}} \cap \widehat{X_{2}}=\varnothing$.

В этом параграфе рассматривается специальньй частный случай, в котором $X_{1}=\Gamma$ - произвольный контур, $\Gamma \notin \mathscr{N}\left(\right.$ т.е. $\left.\mathscr{P}_{n}(\Gamma)=C(\Gamma)\right)$, а $X_{2}=K-$ компакт в $D=D(\Gamma)$ с условием (1.2). Мы получим два достаточных условия, при которых $X=K \cup \Gamma$ удовлетворяет (1.2), а также несколько примеров, для которых это не так. В результате становится ясньм, что основную роль здесь играет положение $K$ относительно немероморфных особых точек (обобщенной) функции Шварца контура $\Gamma$. 
Для дальнейшего нам необходимо выяснить структуру мер, ортогональных пространству $\mathscr{P}_{1}(X)$ на компактах $X$ рассматриваемого вида.

Лемма 4.1. Пусть Г - спрямляемый контур в $\mathbb{C}, K$ - произвольный компакт в $D=D(\Gamma), X=\Gamma \cup K, \mu$ - мера на $K$. Тогда всякая мера $\sigma$, удовлетворяющая условиям

$$
\text { Spt } \sigma \subset X, \quad \sigma_{\mid K}=\mu, \quad \sigma \perp \mathscr{P}_{1}(X),
$$

имеет следующий вид:

$$
\sigma=\mu+\widehat{\mu}(\zeta) d \zeta_{\mid \Gamma}+h(\zeta) d \zeta_{\mid \Gamma}
$$

где $h \in E_{1}(D)$ (класс Смирнова, см. [5; гл. $\left.\left.3, \S 7\right]\right)$, а $h(\zeta)$ - угловые предельнье значения функции $h$ на $\Gamma$.

Обратно, для всякой меры $\mu$ на $K$ и $h \in E_{1}(D)$ мера $\sigma$ (определенная согласно (4.1)) ортогональна $\mathscr{P}_{1}(X)$.

ДокаЗАТЕЛЬСтво. Проверим, что $\rho:=\mu+\widehat{\mu}(\zeta) d \zeta_{\mid \Gamma} \perp \mathscr{P}_{1}(X)$.

Действительно, для всякого $p \in \mathscr{P}_{1}$ имеем:

$$
\begin{aligned}
\int_{X} p(z) d \rho(z) & =\int_{K} p(z) d \mu(z)+\int_{\Gamma} p(\zeta) \widehat{\mu}(\zeta) d \zeta \\
& =\int_{K}\left[p(z)-\frac{1}{2 \pi i} \int_{\Gamma} \frac{p(\zeta) d \zeta}{\zeta-z}\right] d \mu(z)=\int_{K}[p(z)-p(z)] d \mu(z)=0
\end{aligned}
$$

согласно теоремам Фубини и Коши.

Так как носитель меры $\sigma-\rho$ принадлежит $\Gamma$, то сама эта мера ортогональна $\mathscr{P}_{1}(X)$ и $\mathscr{P}_{1}(\Gamma)$ одновременно. Остается воспользоваться обобшенной теоремой Ф. и М. Риссов (см. [5; гл. 3$])$.

Нам потребуется расширить понятие неванлинновского контура применительно к данной задаче.

ОПРЕДЕЛЕниЕ 4.2. Спрямляемый контур $Г$ в $\mathbb{C}$ назовем локально неванлинновским (пишем $\Gamma \in \mathscr{N}_{\text {loc }}$ ), если сушествуют компактное множество $K_{0} \subset D(\Gamma)$ и голоморфные ограниченные функции $u(\cdot)$ и $v(\cdot) \not \equiv 0$ в $D(\Gamma) \backslash K_{0}$ такие, что для п.в. $\zeta \in \Gamma$ выполняется равенство угловых граничных значений

$$
\bar{\zeta}=\frac{u(\zeta)}{v(\zeta)}
$$

Следуюший результат позволяет найти ряд новых нетривиальных примеров множеств, удовлетворяющих (1.2) при $n \geqslant 2$.

ТеОРема 4.3. Пусть $Г \notin \mathscr{N}-$ спрямляемый контур, $K-$ компакт в $D=$ $D(\Gamma)$ с условием (1.2), а $X=\Gamma \cup K$.

(1) Eсли $\Gamma \notin \mathscr{N}_{\text {loc }}$, то $\mathscr{P}_{n}(X)=\mathscr{A}_{n}(X)$ (здесь $n$ такое жсе, как и для $\left.K\right)$.

(2) Пусть $\Gamma \in \mathscr{N}_{\mathrm{loc}}, u, v$ и $K_{0}$ взяты из определения 4.2. Если и/v не может быть продолжено мероморфно из $D \backslash\left(\widehat{K_{0} \cup K}\right)$ на $D \backslash \widehat{K}$, то $\mathscr{P}_{n}(X)=\mathscr{A}_{n}(X)$. 
ДокаЗАТЕЛьство. Положим противное: $\mathscr{P}_{n}(X) \neq \mathscr{A}_{n}(X)$, так что найдется мера $\sigma$ на $X$ такая, что $\sigma \perp \mathscr{P}_{n}(X)$, но $\sigma \not \perp \mathscr{A}_{n}(X)$. Пусть $\mu=\sigma_{\mid K}$, тогда согласно лемме 4.1 имеет место (4.1) с некоторой функцией $h \in E_{1}(D)$. Так как $\bar{z}^{s} \sigma \perp \mathscr{P}_{1}(X)$ при $s=1, \ldots, n-1$, то мы получаем:

$$
\bar{z}^{s} \mu+\bar{\zeta}^{s} \widehat{\mu}(\zeta) d \zeta_{\mid \Gamma}+\bar{\zeta}^{s} h(\zeta) d \zeta_{\mid \Gamma} \perp \mathscr{P}_{1}(X)
$$

Пусть $\mu_{s}=\bar{z}^{s} \mu$. Снова применяя лемму 4.1, получаем, что

$$
\bar{z}^{s} \mu+\widehat{\mu}_{s}(\zeta) d \zeta_{\mid \Gamma} \perp \mathscr{P}_{1}(X)
$$

Следовательно, при $s=1, \ldots, n-1$ имеем:

$$
\left(\bar{\zeta}^{s} \widehat{\mu}(\zeta)-\widehat{\mu_{s}}(\zeta)+\bar{\zeta}^{s} h(\zeta)\right) d \zeta \mid \Gamma \perp \mathscr{P}_{1}(X)
$$

А так как носители этих мер лежат на $\Gamma$, то все они ортогональны также и $\mathscr{P}_{1}(\Gamma)$. По (обобщенной) теореме $\Phi$. и М. Риссов найдутся функции $h_{s} \in E_{1}(D)$ такие, что $\bar{\zeta}^{s} \widehat{\mu}(\zeta)-\widehat{\mu_{s}}(\zeta)+\bar{\zeta}^{s} h(\zeta)=h_{s}(\zeta)$ или, что то же самое,

$$
\bar{\zeta}^{s}(h(\zeta)+\widehat{\mu}(\zeta))=h_{s}(\zeta)+\widehat{\mu_{s}}(\zeta)
$$

$d \zeta_{\mid \Gamma^{-п . в . ~ п р и ~} s=1, \ldots, n-1 .}$

Мы утверждаем, что $h(z)+\widehat{\mu}(z) \not \equiv 0$ в $D \backslash \widehat{K}$ (откуда $h(\zeta)+\widehat{\mu}(\zeta) \neq 0$ п.в. на $\Gamma)$. В самом деле, в противном случае ввиду (4.1) и (4.3) мы бы имели $\mu \perp \mathscr{P}_{n}(X)$. А поскольку Spt $\mu \subset K$, то $\mu \perp \mathscr{P}_{n}(K)=\mathscr{A}_{n}(K), \mu \neq 0$. Откуда с очевидностью $\mu \perp \mathscr{A}_{n}(X)$, что и дает нужное противоречие.

Таким образом, при $s=1, \ldots, n-1$ для п.в. точек $\zeta \in \Gamma$ имеет место равенство угловых предельных значений:

$$
\bar{\zeta}^{s}=\frac{h_{s}(\zeta)+\widehat{\mu_{s}}(\zeta)}{h(\zeta)+\widehat{\mu}(\zeta)}
$$

Пусть $k$ конформно отображает $D_{1}$ на $D$. Согласно $[5 ;$ гл. $3, \S 6]$ функции $h_{1}(k(w)) k^{\prime}(w), h(k(w)) k^{\prime}(w)$ и $k^{\prime}(w)$ принадлежат классу $H_{1}\left(D_{1}\right)$ (а следовательно, и $N\left(D_{1}\right)$ ), поэтому найдутся ограниченные голоморфные в $D$ функции $u_{0}, u_{1}$, $v_{0}$ и $v_{1}$ такие, что $h_{1}=u_{1} / v_{1}$ и $h=u_{0} / v_{0}$. Отсюда для п.в. $\zeta \in \Gamma$ получаем

$$
\bar{\zeta}=\frac{\left(u_{1}(\zeta)+v_{1}(\zeta) \widehat{\mu_{1}}(\zeta)\right) v_{0}(\zeta)}{\left(u_{0}(\zeta)+v_{0}(\zeta) \widehat{\mu}(\zeta)\right) v_{1}(\zeta)}
$$

где числитель и знаменатель в (4.5) голоморфны и ограничены вблизи Г в $D$. Последнее означает, что $\Gamma \in \mathscr{N}_{\text {loc }}$, а это противоречит нашему предположению в (1).

Для доказательства (2) мы используем (4.5) и граничную теорему единственности Лузина-Привалова [5; гл. 4, раздел 2.6], согласно которым $u / v$ должно совпадать с правой частью $(4.5)$ в $D \backslash\left(\widehat{K_{0} \cup K}\right)$ и, следовательно, $u / v$ должно продолжаться по формуле (4.5) мероморфно в $D \backslash \widehat{K}$.

ПРЕДЛОЖЕНИЕ 4.4. В теореме 4.3 условие спрямляемости контура Г $\notin \mathscr{N}$ мохет бить опущено. 
ДоКАЗАТЕЛЬСтво. Во-первых, заметим, что свойство локальной неванлинновости, введенное для спрямляемых контуров, допускает естественное распространение на ограниченные односвязные области $G$ : достаточно в определении 4.2 заменить $D(\Gamma)$ на $G, \Gamma$ на $\partial G$ и понимать равенство (4.2) в смысле конформных отображений (см. (2.1) в определении 2.1).

Пусть $K \subset \Omega$ - компакт с условием (1.2) и $X=\partial \Omega \cup K$. Предположим, что $\mathscr{A}_{n}(X) \neq \mathscr{P}_{n}(X)$ и $\sigma \not \equiv 0$ - такая мера $X$, что $\sigma \perp \mathscr{P}_{n}(X)$, но $\sigma \not \perp \mathscr{A}_{n}(X)$. Пусть $k$ - конформное отображение $D_{1}$ на $\Omega$, продолженное согласно теореме Каратеодори (см. [19; гл. II, раздел С, п. 1]) до гомеоморфизма замкнутых областей $\overline{D_{1}}$ и $\bar{\Omega}$. "Перенесем картинку" из $\bar{\Omega}$ в $\overline{D_{1}}$ с помощью $k^{-1}$. Пусть $K^{\prime}=k^{-1}(K)$ и $X^{\prime}=$ $\Gamma_{1} \cup K^{\prime}$. Определим меру $\sigma^{\prime}$ на $X^{\prime}$ по формуле $\sigma^{\prime}=k^{-1}(\sigma)$, так что

$$
\int f(w) d \sigma^{\prime}(w)=\int f\left(k^{-1}(z)\right) d \sigma(z)
$$

для любой функции $f \in C\left(\overline{D_{1}}\right)$. Покажем, что $\bar{k}^{s} \sigma^{\prime} \perp \mathscr{P}_{1}\left(X^{\prime}\right)$ при $s=0, \ldots, n-1$. В самом деле, для любого $p \in \mathscr{P}_{1}$ имеем

$$
\int_{X^{\prime}} p(w) \overline{k(w)}^{s} d \sigma^{\prime}(w)=\int_{X} p\left(k^{-1}(z)\right) \bar{z}^{s} d \sigma(z)=\lim _{j \rightarrow \infty} \int_{X} q_{j}(z) \bar{z}^{s} d \sigma(z)=0,
$$

где последовательность $\left\{q_{j}\right\} \subset \mathscr{P}_{1}$ выбрана так, что $\left\|q_{j}-p\left(k^{-1}(\cdot)\right)\right\|_{\Omega} \rightarrow 0$ при $j \rightarrow \infty$ (это возможно по теореме Мергеляна).

Остается дословно повторить доказательство теоремы 4.3, заменяя (всюду, где нужно) умножение на $\bar{z}^{s}$ умножением на $\overline{k(w)}$. Так, вместо (4.4) получаем:

$$
\overline{k(t)}^{s}\left(h(t)+\widehat{\mu^{\prime}}(t)\right)=h_{s}(t)+\widehat{\left(\bar{k}^{s} \mu^{\prime}\right)}(t)
$$

при $s=1, \ldots, n-1$ для п.в. точек $t \in \Gamma_{1}$, где $\mu^{\prime}=\sigma_{\mid K^{\prime}}^{\prime}$ и функции $h(\cdot)$ и $h_{s}(\cdot)$ при $s=1, \ldots, n$ принадлежат $H_{1}\left(D_{1}\right)$.

Итак, если $\Gamma$ - какой-либо контур, содержаший аналитически независимые ду-

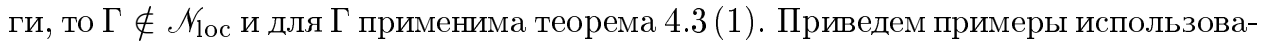
ния теоремы $4.3(2)$.

ПримеР 4.5. Пусть $S$ - функция Шварца (3.3) эллипса (3.2), т.е. $\Gamma=\Gamma_{a b}$. Поскольку полное аналитическое продолжение по Вейерштрассу аналитического элемента $(S, \mathbb{C} \backslash[-c, c])$ имеет только две (конечные) особые точки $\pm c$ - ветвления второго порядка, то по теореме 4.3(2) при всех $n \geqslant 2$ имеет место $\mathscr{P}_{n}(K \cup \Gamma)=$ $\mathscr{A}_{n}(K \cup \Gamma)$ для любого компакта $K$ в $D$ с условием $(1.2)$, у которого $\widehat{K}$ "не соединяет" $c$ и $-c$ (или проще: если $\widehat{K}$ не содержит одну из них). Здесь в качестве $K_{0}$ берется подходящая жорданова кривая в $D(\Gamma)$, соединяющая $c$ и $-c$.

Представляется интересным найти также противоположный пример для этого же Г. Пусть $\gamma$-какая-либо простая аналитическая дуга в $D=D(\Gamma)$, соединяюшая $c$ и $-c$, обладаюшая тем свойством, что ее функция Шварца $\varphi$ (т.е. $\varphi(z)=\bar{z}$ на $\gamma$ ) голоморфна в некоторой окрестности множества $\bar{D}$; например, $\gamma=[-c, c]$, а $\varphi(z)=z$. Мы докажем, что если $K=\gamma$, то $\mathscr{P}_{2}(X) \neq C(X)$, однако $\mathscr{P}_{n}(X)=C(X)$ при $n \geqslant 3$. 
Пусть $\sigma \perp \mathscr{P}_{1}(X), \mu=\sigma_{\mid K}$. По лемме 4.1 найдется $h \in E_{1}(D)$ такая, что

$$
\sigma=\mu+\widehat{\mu}(\zeta) d \zeta_{\mid \Gamma}+h(\zeta) d \zeta_{\mid \Gamma}
$$

Так как $\varphi \in \mathscr{P}_{1}(\bar{D}) \subset \mathscr{P}_{1}(X)$, то $\varphi^{s} \sigma \perp \mathscr{P}_{1}(X)$ при $s=1, \ldots, n-1$. Следовательно, $\sigma \perp \mathscr{P}_{n}(X)$, если и только если $\left(\bar{z}^{s} \sigma-\varphi^{s} \sigma\right) \perp \mathscr{P}_{1}(X)$ или, эквивалентно (поскольку $\bar{z}=\varphi(z)$ на $\gamma$ ),

$$
\left(\bar{\zeta}^{s}-\varphi^{s}(\zeta)\right)\left(\widehat{\mu}(\zeta) d \zeta_{\mid \Gamma}+h(\zeta) d \zeta_{\mid \Gamma}\right) \perp \mathscr{P}_{1}(\Gamma)
$$

при $s=1, \ldots, n-1$.

Таким образом, условие $\mathscr{P}_{n}(X) \neq C(X)$ эквивалентно тому, что найдутся ненулевая мера $\mu$ на $\gamma$ и функции $h \in E_{1}(D)$ и $h_{s} \in E_{1}(D)$ такие, что (ввиду $\bar{\zeta}=S(\zeta)$ на Г):

$$
\left(S^{s}(z)-\varphi^{s}(z)\right)(\widehat{\mu}(z)+h(z))=h_{s}(z), \quad s=1, \ldots, n-1,
$$

в $D \backslash \gamma$ (заметим, что здесь $S$ выбрана голоморфной на $\mathbb{C} \backslash \gamma)$.

Пусть $n \geqslant 3$ и указанные $\mu \neq 0, h, h_{s}$ с условиями (4.6) сушествуют. Как и в доказательстве теоремы $4.3(1)$ получаем, что $h(z)+\widehat{\mu}(z) \not \equiv 0$ в $D \backslash \gamma$, так что $h_{1} \not \equiv 0$ и $h_{2} \not \equiv 0$ в $D$. В то же время из $(4.6)$ следует, что $h_{2}(z)=h_{1}(z)(S(z)+\varphi(z))$, что невозможно. Таким образом, $\mathscr{P}_{n}(X)=C(X)$ при $n \geqslant 3$.

Пусть теперь $n=2$. Найдем нужные $\mu, h, h_{1}$. Элементарные вычисления показывают, что $S(z) \neq \bar{z}$ при $z \in D$ (при $z \in \gamma$ берутся граничные значения функции $S$ на $\gamma$ ). Последнее означает (ввиду $\varphi(z)=\bar{z}$ на $\gamma$ ), что функция $\frac{1}{S(z)-\varphi(z)}$ ограничена вблизи $\gamma$, т.е. $S(z)-\varphi(z)$ имеет только конечное число нулей в $W \backslash \gamma($ где $W-$ некоторая окрестность множества $\bar{D}$, в которой $\varphi$ голоморфна). Возьмем в качестве $h_{1}$ некоторьй полином с такими же нулями (с учетом порядков), как у $S-\varphi$ в $W \backslash \gamma$. Нам надо иметь

$$
\widehat{\mu}(z)=\frac{h_{1}(z)}{S(z)-\varphi(z)}-h(z) .
$$

Пусть $\chi \in C_{0}^{\infty}(W)$ такова, что $\chi=1$ в некоторой окрестности $W_{1}$ множества $\bar{D}$, $W_{1} \subset W$. Определим

$$
\mu=\chi \bar{\partial}\left(\frac{h_{1}}{S-\varphi}\right)
$$

где последнее равенство понимается в обобшенном смысле. Из формулы Коши и хорошо известной конструкции $*$-слабых пределов соответствуюших мер можно показать, что $\mu$ есть ненулевая мера на $\gamma$, абсолютно непрерывная относительно длины на $\gamma$.

Наконец, положим

$$
h=\frac{h_{1}}{S-\varphi}-\widehat{\mu} \text { в } W_{1} .
$$

Тогда $h \in \mathscr{A}_{1}(\bar{D}) \subset E_{1}(D)$. Ввиду $\mathscr{P}_{2}(\gamma)=C(\gamma)$ найдется полином $p \in \mathscr{P}_{2}$ со свойством $\int p d \mu \neq 0$. Теперь возьмем функцию $f=p$ на $K$ и $f(\zeta)=0$ при $\zeta \in \Gamma$. Тогда $f \in C(X)$, но $\int f d \sigma=\int f d \mu \neq 0$. По построению $\sigma \perp \mathscr{P}_{2}(X)$, а значит, $C(X) \neq \mathscr{P}_{2}(X)$. 
Пример 4.6. В этом примере мы покажем, что условие на $X$, достаточное для выполнения (1.2), которое приведено в теореме $4.3(2)$, не является необходимым. Конкретно, (1.2) может выполняться для $X=K \cup \Gamma$, где $\Gamma$ - аналитический контур, у которого функция Шварца $S$ голоморфна в $D \backslash \widehat{K}, K=\widehat{K}$ и $K^{\circ}=\varnothing$.

Пусть $\Gamma$ - образ единичной окружности $\Gamma_{1}$ под действием отображения $z=$ $\exp w$. Для Г имеем $S(z)=\exp (1 / \log z)$, где $\log z$ есть главная ветвь логарифма в правой полуплоскости. Функция $S$ имеет единственную особую точку $z=1$ в $D$, которая является существенно особой, так что $\mathscr{P}_{2}(\Gamma)=C(\Gamma)$. Мы утверждаем, что если $K=\widehat{K}$ имеет плоскую лебегову меру нуль, то $\mathscr{P}_{2}(X)=C(X)$. По теореме 4.3 нам нужно рассмотреть только случай $1 \in K$. Пусть, от противного, $\mathscr{P}_{2}(X) \neq C(X)$. Тогда согласно (4.4) найдутся мера $\mu \neq 0$ на $K$, функции $h$ и $h_{1}$ из $E_{1}(D)$ такие, что

$$
\widehat{(\bar{z} \mu)}-S \widehat{\mu}=S h-h_{1}
$$

в $D \backslash K$. Так как $K$ имеет нулевую лебегову меру, то (4.7) верно в $D \backslash\{1\}$ в обобшенном смысле. Применяя оператор $\bar{\partial}$ к (4.7), мы получаем

$$
\bar{z} \mu-S(z) \mu=0, \quad z \in D \backslash\{1\}
$$

Непосредственно проверяется, что $\bar{z} \neq S(z)$ в $D$, откуда Spt $\mu=\{1\}$. Следовательно,

$$
\widehat{\mu}(z)=\widehat{(\bar{z} \mu)}=\frac{\text { Const }}{z-1},
$$

что противоречит (4.7), ибо для $S$ точка 1 существенно особая. Можно показать, что тот же результат справедлив, если $S$ имеет только конечное число особых точек в $D(\Gamma \notin \mathscr{N})$ и конечное число точек $z \in D$ с условием $\bar{z}=S(z)$.

\section{§5. Аппроксимация бианалитическими полиномами и $\bar{\partial}^{2}-$ задача Дирихле}

Пусть $\Omega$ - произвольная ограниченная область в $\mathbb{C}$ с границей $T$. Определим

$$
\mathscr{S}_{2}(T)=\left\{\varphi \in C(T): \exists f_{\varphi} \in C(\bar{\Omega}), \bar{\partial}^{2} f(z)=0, z \in \Omega,\left.f_{\varphi}\right|_{T}=\varphi\right\}
$$

В стандартной терминологии $\mathscr{S}_{2}(T)$ есть совокупность функций $\varphi \in C(T)$, для которых $\bar{\partial}^{2}$-задача Дирихле разрешима в классическом смысле. Говорят, что $\Omega$ является $\bar{\partial}^{2}$-регулярной, если $\mathscr{S}_{2}(T)=C(T)$, и $\bar{\partial}^{2}$-иррегулярной в противном случае. На самом деле возникает

ЗАДАчА 5.1. Сушествует ли хотя бы одна $\bar{\partial}^{2}$-регулярная область в $\mathbb{C} ?$

Следуюшее предложение подтверждает, что ответ на последний вопрос скорее всего отрицателен.

ПРЕДЛОЖЕНИЕ 5.2. Пусть Г - контур, содержсащий какую-либо аналитическую дугу $\gamma$, тогда область $D=D(\Gamma)$ является $\bar{\partial}^{2}$-иррегулярной. 
ДокАЗАТЕЛЬСТво. Пусть $S$ - функция Шварца дуги $\gamma$, т.е. $S$ голоморфна в некоторой окрестности $U$ дуги $\gamma$ и $\bar{z}=S(z)$ на $\gamma$. Мы можем дополнительно потребовать, чтобы $U \cap D$ было связным. Возьмем какую-либо точку $a \in U \cap D$. Мы утверждаем, что функция $\varphi:=1 /\left.(z-a)\right|_{\Gamma} \notin \mathscr{S}_{2}(\Gamma)$. Пусть, от противного, найдется $f_{\varphi} \in \mathscr{A}_{2}(\bar{D}),\left.f_{\varphi}\right|_{\Gamma}=\varphi$. Тогда $f_{\varphi}(z)=f_{0}(z)+\bar{z} f_{1}(z)$, где $f_{0}$ и $f_{1}$ голоморфны в $D$. Ввиду [3; лемма 3$]$ имеем:

$$
\left|f_{1}(z)\right| \leqslant A \frac{\omega\left(f_{\varphi}, \operatorname{dist}(z, \Gamma)\right)}{\operatorname{dist}(z, \Gamma)}
$$

для всех $z \in D$, где $\omega\left(f_{\varphi}, \cdot\right)$ есть модуль непрерывности функции $f_{\varphi}$ в $\bar{D}$ и $A$ - абсолютная константа. Рассмотрим "когерентную" функцию $f_{\Gamma}(z)=f_{0}(z)+$ $S(z) f_{1}(z)$ (см. [1; с. 53]), голоморфную в $D \cap U$. Из (5.1) получаем

$$
f_{\varphi}(z)-f_{\Gamma}(z)=f_{1}(z)(\bar{z}-S(z)) \rightarrow 0 \text { равномерно при } z \rightarrow \gamma_{0}, \quad z \in D \cap U,
$$

где $\gamma_{0}$ - некоторая невырожденная аналитическая поддуга в $\gamma$. Следовательно, $f_{\Gamma}$ непрерывно продолжается на $D \cup \gamma_{0}, f_{\Gamma}=\varphi$ на $\gamma_{0}$, так что $f_{\Gamma}(z)=1 /(z-a)$ в $D \cap U$, что невозможно.

СлеДСТвИЕ 5.3. (1) Для всякого контура Г справедливо

$$
\mathscr{S}_{2}(\Gamma) \subset \mathscr{P}_{2}(\Gamma) \subset C(\Gamma) .
$$

(2) Для любого $\Gamma \notin \mathscr{N}$, содержащего некоторую аналитическую поддугу,

$$
\mathscr{S}_{2}(\Gamma) \neq \mathscr{P}_{2}(\Gamma)=C(\Gamma) .
$$

ДокАЗАТЕЛЬСтво. Для доказательства (1) пользуемся теоремой 1.3; чтобы установить (2), применяем теорему 2.2 и предложение 5.2 .

Представляется полезньм сравнить (5.2) с соответствующей ситуацией для гармонических функций в шкале равномерной и липшицевых норм. Пусть $\Gamma$ - произвольный контур в $\mathbb{C}$. При любом $q \in[0,1 / 2)$ всякая функция $f \in \operatorname{lip}(q, \Gamma)$ может быть единственным образом продолжена до функции $F \in \operatorname{lip}(q, \bar{D})$, гармонической в $D=D(\Gamma)($ см. [20]), причем $f$ приближаема в $\operatorname{Lip}(q, \Gamma)$-норме на $\Gamma$ гармоническими полиномами (см. [21; следствие 1.2$]$; там же, в [21], можно найти определения пространств $\operatorname{Lip}(q, X)$ и $\operatorname{lip}(q, X), q \in[0,1))$. При $q \in[1 / 2,1)$ существуют контур $\Gamma$, а также функция $f \in \operatorname{lip}(q, \Gamma), \operatorname{Lip}(q, \Gamma)$-приближаемая на $\Gamma$ гармоническими полиномами, для которой соответствуюшее решение $F$ задачи Дирихле не принадлежит $\operatorname{Lip}(q, \bar{D})$ (см. [21; предложение 3.5$])$. Это означает, что ситуация, аналогичная (5.2), для гармонических функций возникает только с порядка гладкости $q=1 / 2$.

В следуюшем результате дается полное описание пространств $\mathscr{S}_{2}(\Gamma)$ и $\mathscr{P}_{2}(\Gamma)$ в специальном случае, когда Г есть аналитический неванлинновский контур.

ПРЕДЛОЖЕНИЕ 5.4. Пусть Г - аналитический неванлинновский контур, $D=D(\Gamma)$, a $S=u / v-$ функция Шварца контура $\Gamma$ (мь вправе считать, что и и $v$ голоморфны на открытом множестве $U$, содержащем $\bar{D}$, причем и и v не имеют общих нулей в $U)$. Тогда

$$
\mathscr{S}_{2}(\Gamma)=\mathscr{P}_{2}(\Gamma)=\left\{\varphi \in C(\Gamma): P[\varphi v] \in \mathscr{A}_{1}(\bar{D})\right\},
$$

әде $P$ - оператор Пуассона для области D. 
ДоКАЗАТЕЛЬСтво. Обозначим через $B(Г)$ правую часть в (5.3). По следствию 5.3 достаточно показать, что $\mathscr{P}_{2}(\Gamma) \subset B(\Gamma)$ и $B(\Gamma) \subset \mathscr{S}_{2}(\Gamma)$. Пусть $\varphi \in$ $\mathscr{P}_{2}(\Gamma)$. Тогда найдутся последовательности $\left\{p_{m}(z)\right\}_{m=1}^{\infty} \subset \mathscr{P}_{1}$ и $\left\{q_{m}(z)\right\}_{m=1}^{\infty} \subset$ $\mathscr{P}_{1}$ такие, что $\varphi=\lim _{m \rightarrow \infty}\left(p_{m}+\bar{z} q_{m}\right)$ равномерно на $\Gamma$. Отсюда вытекает, что $\varphi v=\lim _{m \rightarrow \infty}\left(v p_{m}+u q_{m}\right)$ равномерно на $Г$ и, следовательно, по принципу максимума модуля $\left\{v p_{m}+u q_{m}\right\}$ сходится равномерно на $\bar{D}$ к функции $f \in \mathscr{A}_{1}(\bar{D})$. Откуда $P[\varphi v]=f$.

Теперь докажем другое включение. Согласно [22; с. 329] найдутся функции $e_{1}(\cdot)$ и $e_{2}(\cdot)$, голоморфные в $U$, с условием $e_{1} v+e_{2} u=1$. Отсюда для всякой функции $f \in \mathscr{A}_{1}(\bar{D})$ выполнено $f=v f_{1}+u f_{2}$, где $f_{1}=f e_{1} \in \mathscr{A}_{1}(\bar{D})$ и $f_{2}=f e_{2} \in$ $\mathscr{A}_{1}(\bar{D})$.

Теперь возьмем любую функцию $\varphi \in B(\Gamma)$ и рассмотрим $f=P[\varphi v]$. Согласно приведенньм аргументам

$$
P[\varphi v]=v f_{1}+u f_{2} \text { в } \bar{D},
$$

поэтому $\varphi=f_{1}+f_{2}(u / v)=f_{1}+f_{2} \bar{z}$ на $\Gamma$ при $f_{1}, f_{2} \in \mathscr{A}_{1}(\bar{D})$. Следовательно, $\varphi \in \mathscr{S}_{2}(\Gamma)$. Этим завершается доказательство (5.3).

Следующая теорема указывает также на отсутствие эквивалентности между условием приближаемости (1.2) при $X=\partial \Omega$ для области Каратеодори $\Omega$ и свойством единственности решений $\bar{\partial}^{2}$-задачи Дирихле в $\Omega$.

Теорема 5.5. (1) Если $\Omega \in \mathscr{N}$ - область Каратеодори, то найдется такая функиия $f \in \mathscr{A}_{2}(\bar{\Omega})$, ито $\left.f\right|_{\partial \Omega}=0$, но $f \not \equiv 0$ в $\Omega$.

(2) Существуют спрямляемый контур $\Gamma \notin \mathscr{N}_{10 с}$ и функиия $f$ с теми же свойствами, что и в (1); указанный контур может даже быть аналитическим всюду, за исключением одной точки.

ЗАмечАниЕ 5.6. Согласно [1; теорема 3.8$]$ каждый аналитический контур $\Gamma \notin \mathscr{N}$ обладает свойством единственности, т.е. из $f \in \mathscr{A}_{2}(\bar{D}), D=D(\Gamma)$, и $\left.f\right|_{\Gamma}=0$ вытекает $f \equiv 0$ в $D$.

ДоказатеЛЬСтво теоремы $5.5(1)$. Пусть $\Omega \in \mathscr{N}$. Так же как в доказательстве теоремы 2.2 , мы можем найти ненулевую меру $\mu$ на $\partial \Omega$ такую, что $\mu \perp \mathscr{R}_{2}(\partial \Omega, \bar{\Omega})$. Согласно [7; лемма 9] мера $\mu$ не имеет атомов. Рассмотрим функцию

$$
\Psi(z)=\frac{1}{2 \pi i} \int_{\partial \Omega} \frac{\bar{\zeta}-\bar{z}}{z-\zeta} d \mu(\zeta),
$$

которая (в силу аргументов, приведенных в доказательстве утверждения (1) теоремы 2.2) всюду определена, непрерывна и обрашается в нуль вне $\Omega$. Так как $\mu \not \equiv 0$, то $\Psi \not \equiv 0$, т.е. функция $\Psi(\cdot)$ полностью удовлетворяет условиям п. (1) теоремы.

ДОКАЗАТЕЛЬСТВо ПРЕДЛОЖЕНИЯ 3.3. Пусть $\Gamma \in \mathscr{N}$ спрямляем, а $u$ и $v$ взяты из определения 2.1. По формуле Коши, которая остается справедливой для функций класса $H_{\infty}(D)$, имеем:

$$
u(z)-\bar{z} v(z)=\frac{1}{2 \pi i} \int_{\Gamma} \frac{\bar{\zeta}-\bar{z}}{\zeta-z} v(\zeta) d \zeta, \quad z \in D,
$$

так что, как и чуть вьше, остается заметить, что мера $v d \zeta_{\mid \Gamma}$ конечна и не имеет атомов. 
ДОКАЗАТЕЛЬСТВо ТЕОРЕМЫ 5.5 (2). Здесь мы будем следовать идее Мазалова [16], модифицируя его конструкцию нигде не аналитического неванлинновского контура.

Возьмем какую-либо последовательность различных точек $\left\{a_{l}\right\}$ таких, что $2 / 3<\left|a_{l}\right|<1$ и $\left|a_{l}\right| \nearrow 1$ при $l \rightarrow+\infty$, и последовательность положительных чисел $\left\{r_{l}\right\}$, которые (обе) будут конкретизированы позже. Сейчас мы только потребуем, чтобы все круги $B_{l}=B\left(a_{l}, r_{l}\right), l \geqslant 1$, были попарно внешними и $r_{l}<d_{l} / 2$, где $d_{l}=1-\left|a_{l}\right|$. В частности, $\bigcup_{l=1}^{\infty} B_{l} \subset\{w: 1 / 2<|w|<1\}$.

Теперь возьмем последовательность $\left\{\varepsilon_{l}\right\}, \varepsilon_{l}>0$, с условием

$$
\sum_{l=1}^{\infty} \frac{\varepsilon_{l}}{r_{l}} \leqslant 1 \text {, }
$$

гарантируюшим, что функция

$$
g(w)=\frac{1}{w}+\sum_{l=1}^{\infty} \frac{\varepsilon_{l}}{w-a_{l}}
$$

мероморфна в $D_{1}$, голоморфна в $\overline{\mathbb{C}} \backslash \overline{D_{1}}$ и непрерывна на $\overline{\mathbb{C}} \backslash D_{1}$. Более того,

$$
\begin{aligned}
& |g(w)| \leqslant \frac{1}{|w|}+1 \quad \text { при } \quad w \in \mathbb{C} \backslash \bigcup_{n=1}^{\infty} B_{l}, \\
& |g(w)| \leqslant 3+\frac{\varepsilon_{j}}{\left|w-a_{j}\right|} \quad \text { при } \quad w \in B_{j}, \quad j=1,2, \ldots .
\end{aligned}
$$

Введем $k(w):=g_{*}(1 / w)$ и оценим $|g(w)-\overline{k(w)}|$ при $1 / 2 \leqslant|w|<1, w \notin \bigcup_{l=1}^{\infty} B_{l}$ :

$$
\begin{aligned}
|g(w)-\overline{k(w)}| & \leqslant\left|\frac{1}{w}-\bar{w}\right|+\sum_{l=1}^{\infty} \frac{\varepsilon_{l}\left(1-|w|^{2}\right)}{\left|w-a_{l}\right|\left|1-a_{l} \bar{w}\right|} \\
& \leqslant 3(1-|w|)+\sum_{l=1}^{\infty} \frac{2 \varepsilon_{l}(1-|w|)}{r_{l} d_{l}} \leqslant 5(1-|w|) .
\end{aligned}
$$

Последнее выполняется при дополнительном требовании

$$
\sum_{l=1}^{\infty} \frac{\varepsilon_{l}}{r_{l} d_{l}} \leqslant 1
$$

которое включает (5.4). Ввиду

$$
k(w)=w-\sum_{l=1}^{\infty} \frac{\varepsilon_{l} w}{\bar{a}_{l}\left(w-a_{l}^{*}\right)}, \quad a_{l}^{*}=\frac{1}{\bar{a}_{l}},
$$

имеем

$$
k^{\prime}(w)=1+\sum_{l=1}^{\infty} \frac{\varepsilon_{l}}{\bar{a}_{l}^{2}\left(w-a_{l}^{*}\right)^{2}} .
$$

Из (5.7) следует, что последняя (производная) функция действительно существует в $D_{1}$, причем $k$ задает конформное отображение из $D_{1}$ на некоторую жорданову область с гладкой границей, ибо при $|w| \leqslant 1$ имеет место

$$
\sum_{l=1}^{\infty} \frac{\varepsilon_{l}}{\left|a_{l}\right|^{2}\left|w-a_{l}^{*}\right|^{2}} \leqslant \sum_{l=1}^{\infty} \frac{\varepsilon_{l}}{d_{l}^{2}} \leqslant \frac{1}{2} .
$$

Нам необходимо следуюшее утверждение. 
ЛЕмма 5.7. Существует голоморфная функция $h$ в $D_{1}, h \neq \equiv 0$, со следующ,ими свойствами:

$$
|h(w)|(1-|w|)=\eta(w) \rightarrow 0 \quad n p u \quad|w| \rightarrow 1,
$$

ее мнолсество нулей $\left\{a_{l}\right\}_{l=0}^{\infty}\left(a_{0}=0,2 / 3<\left|a_{l}\right|<1\right.$ при $\left.l \geqslant 1\right)$ не удовлетворяет условию Бляшке, т.е.

$$
\sum_{l=1}^{\infty}\left(1-\left|a_{l}\right|\right)=+\infty,
$$

u, более того, $\lim _{l \rightarrow \infty} a_{l}=1$.

Сначала мы продолжим и завершим доказательство теоремы 5.5; лемма 5.7 будет доказана чуть позже.

Сейчас мы фиксируем $h$ и соответствуюшую $\left\{a_{l}\right\}_{l=0}^{\infty}$ из леммы 5.7. Если

$$
\lambda_{l}=\lim _{z \rightarrow a_{l}} \frac{h(w)}{w-a_{l}},
$$

то мы можем найти такие $\rho_{l}>0$, что при $\left|w-a_{l}\right| \leqslant \rho_{l}$ имеет место

$$
|h(w)| \leqslant\left(\left|\lambda_{l}\right|+1\right)\left|w-a_{l}\right| \leqslant \frac{1}{l}, \quad l \geqslant 1 .
$$

Теперь мы уже можем конкретизировать $\left\{r_{l}\right\}$, которые должны только удовлетворять приведенньм выше (непосредственно перед (5.4)) требованиям и, дополнительно, условиям $r_{l} \leqslant \rho_{l}$ при $l \geqslant 1$. Рассмотрим в $D_{1}$ функцию

$$
F(w)=h(w)(g(w)-\overline{k(w)}),
$$

где $\varepsilon_{l}$ в (5.5) выбраны с условием (5.7).

При $w \in B_{l}$ находим:

$$
\begin{aligned}
|F(w)| & \leqslant|h(w)||g(w)|+|h(w)||k(w)| \\
& \leqslant|h(w)|\left(3+\frac{\varepsilon_{l}}{\left|w-a_{l}\right|}\right)+2|h(w)| \leqslant \frac{5}{l}+\varepsilon_{l}\left(\left|\lambda_{l}\right|+1\right) .
\end{aligned}
$$

Окончательное условие на $\varepsilon_{l}$, дополнительное к (5.7), таково: $\left(\left|\lambda_{l}\right|+1\right) \varepsilon_{l} \leqslant 1 / l$ при $l \geqslant 1$. Из него мы получаем

$$
|F(w)| \leqslant \frac{6}{l}, \quad w \in B_{l} .
$$

Но в $D_{1} \backslash \bigcup_{l=1}^{\infty} B_{l}$, пользуясь (5.6) и (5.8) (также ввиду $h(0)=0$ ), мы имеем

$$
|F(w)| \leqslant \frac{A(1-|w|) \eta(w)}{(1-|w|)}=A \eta(w) \rightarrow 0 \text { при }|w| \rightarrow 1 .
$$

Таким образом, $F$ непрерывно продолжается на $\overline{D_{1}},\left.F\right|_{\Gamma_{1}}=0$, но $F \not \equiv 0$ в $D_{1}$. Теперь положим $\Gamma=k\left(\Gamma_{1}\right), D=D(\Gamma)$. Искомая функция $f \in \mathscr{A}_{2}(\bar{D})$ имеет вид:

$$
f(z)=F\left(k^{-1}(z)\right)=h\left(k^{-1}(z)\right)\left(g\left(k^{-1}(z)\right)-\bar{z}\right) .
$$


Пусть $\Gamma_{1}^{\prime}=\Gamma_{1} \backslash\{1\}$ и $\Gamma^{\prime}=k\left(\Gamma_{1}^{\prime}\right)$. Нетрудно проверить, что $k$ на самом деле конформно в некоторой окрестности множества $\overline{D_{1}} \backslash\{1\}$, т.е. $\Gamma^{\prime}$ есть открытая аналитическая дуга. Функция $g_{1}(z)=g\left(k^{-1}(z)\right)$ мероморфна в $D$, непрерывна на $D \cup \Gamma^{\prime}$, а также $g_{1}(\zeta)=\bar{\zeta}$ на $\Gamma^{\prime}$, поскольку $g$ непрерывна на $\overline{D_{1}} \backslash\{1\}$ и $g=\bar{k}$ на $\Gamma_{1}^{\prime}$. Если, от противного, $\Gamma \in \mathscr{N}_{1 \text { ос }}$, т.е. найдутся $u, v$ и $K_{0}$ как в определении 2.1 , то по теореме Лузина-Привалова имеем $g_{1}=u / v$ всюду в $D \backslash K_{0}$, а это означает, что $v\left(k\left(a_{l}\right)\right)=0$ для всех достаточно больших $l$. Последнее, однако, невозможно, поскольку $v \circ k$ ограничена и голоморфна в $D_{1} \backslash k^{-1}\left(K_{0}\right)$ и, следовательно, из хорошо известных свойств конформных отображений получаем, что $\left\{a_{l}\right\}$ должна удовлетворять условию Бляшке (см. [19; гл. IV, раздел А, п. 1]), что противоречит (5.9).

ДоКАЗАТЕЛЬСТво ЛЕМмЫ 5.7. Рассмотрим функцию

$$
h_{0}(w)=\prod_{m=1}^{\infty}\left(1+\alpha w^{\beta^{m}}\right), \quad w \in D_{1},
$$

где $\alpha>1$ и $\beta \geqslant 2$ целое. В [23; с. 699, случай (4.9)] приведены достаточные условия на $\alpha$ и $\beta$ (например, далее мы берем $\alpha=2, \beta=6$ ), при которых $h_{0}$ принадлежит пространству Бергмана $A^{2}\left(D_{1}\right)$ (т.е. пространству всех голоморфных функций $f$ в $D_{1}$, для которых $|f|^{2}$ интегрируема по плошади в $\left.D_{1}\right)$.

Следовательно, множество $W_{0}$, состоящее из всех корней порядков $6^{m}$ из $-1 / 2$ при $m=1,2, \ldots$, является нулевым множеством для класса $A^{2}\left(D_{1}\right)$. Возьмем

$$
W=\left\{w \in W_{0}: \text { сушествует } m \geqslant 1 \text { такое, что } w^{6^{m}}=-\frac{1}{2}, 0<\arg w<\frac{2 \pi}{m}\right\} \text {. }
$$

Пусть теперь $\left\{a_{l}\right\}$ есть какая-либо последовательность, нумеруюшая точки из $W$ таким образом, что $\left|a_{l}\right| \nearrow 1$ при $l \rightarrow+\infty$. Согласно [23; теорема 3$]$ всякое непустое подмножество любого нулевого множества для $A^{2}\left(D_{1}\right)$ снова является нулевьм множеством для $A^{2}\left(D_{1}\right)$. Поэтому найдется функция $h \in A^{2}\left(D_{1}\right), h \not \equiv 0$, такая, что $h\left(a_{l}\right)=0$ при $l \geqslant 1$ и $h(0)=0$.

Чтобы установить (5.8), достаточно воспользоваться теоремой о среднем и неравенством Шварца:

$$
\pi r^{2}|h(w)|=\left|\int_{B(w, r)} h(z) d x d y\right| \leqslant r \pi^{1 / 2}\left(\int_{B(w, r)}|h(z)|^{2} d x d y\right)^{1 / 2},
$$

где $r=1-|w|$. Откуда

$$
|h(w)|(1-|w|) \leqslant\left(\int_{\{1-2 r<|z|<1\}}|h(z)|^{2} d x d y\right)^{1 / 2} \rightarrow 0 \text { при } r \rightarrow 0
$$

по теореме Лебега об ограниченной сходимости.

Остается доказать, что множество нулей функции $h$ удовлетворяет (5.9). Действительно, если $a_{l} \in W$ является корнем степени $6^{m}$ из $-1 / 2$, то $1-\left|a_{l}\right|=1-$ $\exp \left(-(\log 2) / 6^{m}\right)$. Так как последнее число сравнимо с $(\log 2) / 6^{m}$ и всего имеется порядка $6^{m} / m$ таких корней $a_{l} \in W$, то справедливо (5.9). Очевидно также, что $\lim _{l \rightarrow \infty} a_{l}=1$. Лемма 5.7 и теорема 5.5 доказаны. 
ПримеР 5.8. Теперь мы можем привести пример спрямляемого контура $\Gamma \in \mathscr{N}$ такого, что соответствуюшие функции $u$ и $v$ из определения 2.1 не могут быть (обе из них) взяты из $\mathscr{A}_{1}(\bar{D}), D=D(\Gamma)$.

Рассмотрим доказательство теоремы $5.5(2)$, но теперь вместо функции $h$ из леммы 5.7 мы будем использовать произведение Бляшке $h_{1}$ с тем свойством, что множество предельных точек его нулей $\left\{a_{l}\right\}\left(a_{0}=0,2 / 3<\left|a_{l}\right|<1\right.$ при $l \geqslant 1$, каждый $a_{l}$ есть простой нуль для $\left.h_{1}\right)$ содержит некоторый невырожденный интервал в $\Gamma_{1}$. Как раньше, мы можем выбрать $\left\{r_{l}\right\}$ и $\left\{\varepsilon_{l}\right\}$ так, что функция

$$
F(w)=h_{1}(w)(g(w)-\overline{k(w)})
$$

имеет непрерьвное продолжение на $\overline{D_{1}}$ с условием $\left.F\right|_{\Gamma_{1}}=0$ и $k$ отображает конформно $D_{1}$ на некоторую жорданову область $D$ с гладкой границей $\Gamma$.

По принципу максимума $h_{1} g \in H_{\infty}\left(D_{1}\right)$. Так как угловые пределы функции $h_{1}$ равны по модулю единище п.в. на $\Gamma_{1}$ (см. $\left[22 ;\right.$ c. 335]), то $g(w)$ для п.в. $t \in \Gamma_{1}$ имеет угловые пределы, равные $\overline{k(t)}$. Положим $u(z)=h_{1}\left(k^{-1}(z)\right) g\left(k^{-1}(z)\right), v(z)=$ $h_{1}\left(k^{-1}(z)\right)$, так что $\bar{\zeta}=u(\zeta) / v(\zeta)$ в смысле угловых предельных значений п.в. на $\Gamma$, откуда $\Gamma \in \mathscr{N}$. Пусть, от противного, мы можем найти некоторые ограниченные голоморфные функции $u_{0}$ и $v_{0}$ в $D$ такие, что $u_{0}$ или $v_{0}$ принадлежит $\mathscr{A}_{1}(\bar{D})$ и $\bar{\zeta}=$ $u_{0}(\zeta) / v_{0}(\zeta)$ п.в. на $\Gamma$. Тогда $u v_{0}=u_{0} v$ в $D$. Так как $v\left(k\left(a_{l}\right)\right)=0$, а $u\left(k\left(a_{l}\right)\right) \neq 0$, мы имеем $v_{0}\left(k\left(a_{l}\right)\right)=0$ при $l \geqslant 1$, так что $v_{0}$ не может быть в $\mathscr{A}_{1}(\bar{D})$. Но если $u_{0} \in \mathscr{A}_{1}(\bar{D})$, то $v_{0}(\zeta)=u_{0}(\zeta) / \bar{\zeta}$ непрерьвна на $\Gamma_{1}$ (мы предположили, что $0 \notin \Gamma$ ), однако тогда $v_{0}$ должна принадлежать $\mathscr{A}_{1}(\bar{D})$, что невозможно. Доказательство завершено.

В заключение отметим, что не существует ограниченной односвязной области $\Omega$ такой, что для $\Omega$ выполнены одновременно свойства общей разрешимости и единственности решения $\bar{\partial}^{2}$-задачи Дирихле.

В самом деле, пусть, от противного, существует такая область $\Omega$. Возьмем $z_{0} \in \Omega$ и рассмотрим функцию $\varphi(z)=\left.\frac{1}{z-z_{0}}\right|_{\partial \Omega} \in C(\partial \Omega)$. В силу свойства разрешимости сушествует функция $f_{\varphi} \in C(\bar{\Omega})$, бианалитическая в $\Omega$, с условием $\left.f_{\varphi}\right|_{\partial \Omega}=\varphi$. Тогда $\left(z-z_{0}\right) f_{\varphi}(z)-1=0$ на $\partial \Omega$. Так как выполнено свойство единственности, имеем $\left(z-z_{0}\right) f_{\varphi}(z)-1=0$ в $\Omega$. А это неверно, например в точке $z_{0}$.

\section{Список литературы}

1. Balk M. B. Polyanalytic functions. Berlin: Akademie-Verlag, 1991. (Math. Res. V. 63.)

2. Мергелян С. Н. Равномерное приближение функций комплексного переменного // УМН. 1952. T. 7. № 2. C. 31-122.

3. Carmona J. J. Mergelyan approximation theorem for rational modules // J. Approx. Theory. 1985. V. 44. P. 113-126.

4. Федоровский $K$. Ю. О равномерных приближениях функций $n$-аналитическими полиномами на спрямляемых контурах в $\mathbb{C} / /$ Матем. заметки. 1996. Т. 59. № 4. С. 604-610.

5. Привалов И. И. Граничные свойства аналитических функций. М.-Л.: Гостехиздат, 1950.

6. Лаврентьев M. A. О функциях комплексного переменного, представимых рядами полиномов // Избранные труды. Матем. и мех. М.: Наука, 1990. С. 149-185.

4 Математический сборник, т. 193, №10 
7. Бишоп Э. Граничные меры аналитических диффференциалов // Некоторые вопросы теории приближений. М.: ИЛ, 1963. С. 87-100.

8. Carathèodory C. Conformal representations. New York: Dover Publ., 1998.

9. Витушкин А. Г. Аналитическая емкость множеств в задачах теории приближений // УМН. 1967. Т. 22. №6. С. 141-199.

10. Парамонов П. В., Федоровский $K$. Ю. О равномерной и $C^{1}$-приближаемости функций на компактах в $\mathbb{R}^{2}$ решениями эллиптических уравнений второго порядка // Матем. сб. 1999. Т. 190. № 2. С. $123-144$.

11. Trent T., Wang J. L. Uniform approximation by rational modules on nowhere dense sets // Proc. Amer. Math. Soc. 1981. V. 81. P. 62-64.

12. Wang J. L. A localization operator for rational modules // Rocky Mountain J. Math. 1989. V. 19. № 4. P. 999-1002.

13. Verdera $J$. On the uniform approximation problem for the square of the Cauchy-Riemann operator // Pacific J. Math. 1993. V. 159. P. 379-396.

14. Fedorovski K. Yu. On uniform approximation by polyanalytic polynomials on compact subsets of the plane // Ann. Univ. Mariae Curie-Skłodowska Sect. A. 1999. V. 53. № 3 . P. 27-39.

15. Davis $P$. The Schwarz function and its applications. Washington, DC: The Mathematical Association of America, 1974. (Carus Math. Monogr. V. 17.)

16. Мазалов $M . \mathcal{A}$. Пример непостоянной бианалитической функции, обращающейся в нуль всюду на нигде не аналитической границе // Матем. заметки. 1997. Т. 62. № 3-4. C. 524-526.

17. Бовен А., Парамонов П. В. Аппроксимация мероморфньми и целыми решениями эллиптических уравнений в банаховых пространствах распределений // Матем. сб. 1998. T. 189. № 4. C. 3-24.

18. Douglas R. G., Shapiro H. S., Shields A. L. Cyclic vectors and invariant subspaces for the backward shift operator // Ann. Inst. Fourier (Grenoble). 1970. V. 20. № 1. P. 37-76.

19. Кусис П. Введение в теорию пространств $H_{p}$. М.: Мир, 1984.

20. Johnston E. H. The boundary modulus of continuity of harmonic functions // Pacific J. Math. 1980. V. 90. P. 87-98.

21. Парамонов П. В. $C^{m}$-приближения гармоническими полиномами на компактных множествах в $\mathbb{R}^{n}$ // Матем. сб. 1993. Т. 184. № 2. С. 105-128.

22. Rudin W. Real and complex analysis. New York: McGraw-Hill, 1987.

23. Horowitz Ch. Zeros of functions in the Bergman spaces // Duke Math. J. 1974. V. 41. P. $693-710$.

Автономный университет г. Барселона (Испания);

Поступила в редакцию

Московский государственный университет им. М.В. Ломоносова;

26.02 .2002

Государственный университет управления

E-mail: jcar@mat.uab.es, petr@paramonov.msk.ru, const@fedorovski.mccme.ru 